TRANSACTIONS OF THE

AMERICAN MATHEMATICAL SOCIETY

Volume 351, Number 12, Pages 5027-5049

S 0002-9947(99)02310-7

Article electronically published on August 10, 1999

\title{
WHEN ALMOST MULTIPLICATIVE MORPHISMS ARE CLOSE TO HOMOMORPHISMS
}

\author{
HUAXIN LIN
}

\begin{abstract}
It is shown that approximately multiplicative contractive positive morphisms from $C(X)$ (with $\operatorname{dim} X \leq 2$ ) into a simple $C^{*}$-algebra $A$ of real rank zero and of stable rank one are close to homomorphisms, provided that certain $K$-theoretical obstacles vanish. As a corollary we show that a homomorphism $h: C(X) \rightarrow A$ is approximated by homomorphisms with finite dimensional range, if $h$ gives no $K$-theoretical obstacle.
\end{abstract}

\section{INTRODUCTION}

Let $X$ be a compact metric space and let $A$ be a $C^{*}$-algebra. A contractive positive linear map $\psi: C(X) \rightarrow A$ is said to be $\delta$ - $\mathcal{F}$-multiplicative if

$$
\|\psi(f g)-\psi(f) \psi(g)\|<\delta
$$

for all $f \in \mathcal{F}$. A homomorphism is certainly $\delta$ - $\mathcal{F}$-multiplicative. The problem when an almost multiplicative contractive positive linear morphism is close to a homomorphism has been studied for a long time and recently there has been some important progress. A classical problem is whether for any $\varepsilon>0$ there is $\delta>0$ such that for any $n$ and any pair of selfadjoint matrices $x, y \in M_{n}(\mathbf{C})$ such that $\|x\|,\|y\| \leq 1$ and $\|x y-y x\|<\delta$, there exists a commuting pair $x^{\prime}, y^{\prime} \in M_{n}(\mathbf{C})$ of selfadjoint matrices with $\left\|x^{\prime}-x\right\|+\left\|y-y^{\prime}\right\|<\varepsilon$. It was an old open problem for decades in linear algebra and operator theory which was solved affirmatively recently (see $[\operatorname{Ln} 7])$. This result is equivalent to the following: For any $\varepsilon>0$ and any finite subset $\mathcal{F} \in C(D)$, where $D$ is the unit disk, there is $\delta>0$ and a finite subset $\mathcal{G} \in C(D)$ such that for any finite-dimensional $C^{*}$-algebra $A$ and any $\delta$ $\mathcal{G}$-multiplicative contractive positive linear morphism $\psi: C(D) \rightarrow A$, there is a homomorphism $h: C(D) \rightarrow A$ (with finite-dimensional range) such that

$$
\|\psi(f)-h(f)\|<\varepsilon \text { for all } f \in \mathcal{F} .
$$

The problem mentioned above appears in many different areas of mathematics, e.g., linear algebras, operator theory, as well as in the study of $C^{*}$-algebras.

In general, a $\delta$ - $\mathcal{G}$-multiplicative contractive positive linear morphism is not close to a homomorphism no matter how small $\delta$ is and how large $\mathcal{G}$ is. This was first discovered by D. Voiculescu ( see $[\mathrm{V}]$ ). $K$-theoretical obstacle was later explained by $\mathrm{T}$. Loring (see [Lr2]). Therefore, what we are hoping for is that a $\delta$-G-multiplicative contractive positive linear morphism is close to a homomorphism, provided that

Received by the editors April 10, 1997.

1991 Mathematics Subject Classification. Primary 46L05; Secondary 46L80.

Research partially supported by NSF grant DMS 9531776 .

(C)1999 American Mathematical Society 
$\delta$ is sufficiently small and $\mathcal{G}$ is sufficiently large and that a $K$-theoretical obstacle vanishes. Since every compact metric space $X$ is a subspace of a contractible space $\Omega$, a contractive positive linear morphism $\psi: C(X) \rightarrow A$ can always be viewed as a contractive positive linear morphism from $C(\Omega)$ into $A$. Therefore, some injectivity condition has to be imposed so that we know which obstacle has to vanish.

With the restriction that $A$ is a unital simple $C^{*}$-algebra of real rank zero, stable rank one and unique normalized quasi-trace, it is shown in [GL2] that, if $\operatorname{dim}(X) \leq 2$, an approximately injective and sufficiently multiplicative contractive positive linear morphism $L: C(X) \rightarrow A$ is close to a homomorphism $h: C(X) \rightarrow A$ with finite dimensional range, provided that a $K$-theoretical obstacle vanishes. It is also shown (in [GL2]) that if $X$ is a finite CW complex with dimension greater than 2 , an approximately injective and $\delta$ - $\mathcal{G}$-multiplicative contractive positive linear morphism may not be close to any homomorphism even if all reasonable $K$-theoretical obstructions disappear, no matter how small $\delta$ is and how large $\mathcal{G}$ is. However, for higher dimensional spaces, with an additional injectivity condition, an affirmative solution to the problem can be found in [GL1]. On the other hand, we showed in [Ln11] that, for a given $\varepsilon>0$ and a given finite subset $\mathcal{F} \subset C(X)$, there exist $\delta>0$ and a finite subset $\mathcal{G} \subset C(X)$ such that, for any unital purely infinite simple $C^{*}$-algebra $A$ and any $\delta$ - $\mathcal{G}$-multiplicative contractive positive linear morphism $L: C(X) \rightarrow A$, there is a homomorphism $h: C(X) \rightarrow A$ such that

$$
\|L(f)-h(f)\|<\varepsilon
$$

for all $f \in C(X)$. It is the assumption that $A$ is finite that creates many obstacles as well as technical problems. The above mentioned results about almost multiplicative morphisms have many applications including those to classification of $C^{*}$-algebras of real rank zero and to $C^{*}$-algebra extension theory (see [EGLP], [Ln4], [Ln5], [Ln7], [LP1], [GL1], [Ln13]).

In this paper, we consider a class of simple $C^{*}$-algebras of real rank zero (denoted by $\mathbf{B})$ which consists of all purely infinite simple $C^{*}$-algebras as well as all simple $C^{*}$-algebras of real rank zero and stable rank one. It should be noted that there is no known example of a simple $C^{*}$-algebra of real rank zero which is neither purely infinite nor stable rank one. We will study almost multiplicative contractive positive linear morphisms from $C(X)$ into $C^{*}$-algebras in $\mathbf{B}$, with $\operatorname{dim}(X) \leq 2$. Roughly, the main result of this paper is that, with the above $X$, a $\delta$ - $\mathcal{G}$-multiplicative contractive positive linear morphism from $C(X)$ into any $A \in \mathbf{B}$ is close to a homomorphism, if a $K$-theoretical obstacle vanishes (see, for example, 1.12 and 2.5).

Here are some conventions which are needed in the rest of this paper.

Definition 0.1. Let $\psi: C(X) \rightarrow C$ be a homomorphism, where $C$ is a $C^{*}$-algebra. Let $\Omega$ be the compact subset such that

$$
\operatorname{ker}(\psi)=\{f \in C(X): f(\xi)=0 \text { for all } \xi \in \Omega\} .
$$

We will denote $\Omega$ by $s p(\psi)$.

Definition $\mathbf{0 . 2}$ (cf. 1.2 of [LP1]). Let $\psi$ be a contractive positive linear map from $C(X)$ to a $C^{*}$-algebra $A$, where $X$ is a compact metric space. Fix a finite subset $\mathcal{F}$ contained in the unit ball of $C(X)$. For $\varepsilon>0$, we denote by $\Sigma_{\varepsilon}(\psi, \mathcal{F})$ (or simply $\left.\Sigma_{\varepsilon}(\psi)\right)$ the closure of the set of those points $\lambda \in X$ for which there is a nonzero hereditary $C^{*}$-subalgebra $B$ of $A$ satisfying

$$
\|(f(\lambda)-\psi(f)) b\|<\varepsilon \quad \text { and } \quad\|b(f(\lambda)-\psi(f))\|<\varepsilon
$$


for $f \in \mathcal{F}$ and $b \in B$ with $\|b\| \leq 1$. Note that if $\varepsilon<\sigma$, then $\Sigma_{\varepsilon}(\psi) \subset \Sigma_{\sigma}(\psi)$.

For a $\delta$-G-multiplicative contractive positive linear morphism $\psi$ and a subset $\mathcal{F} \subset \mathcal{G}$, we say $\psi$ is $\sigma$-injective with respect to $\delta$ and $\mathcal{F}$, or $\sigma$ - $\mathcal{F}$-injective, if $\Sigma_{\delta}(\psi, \mathcal{F})$ is $\sigma$-dense in $X$. If $\psi$ is a homomorphism, then $\psi$ is $\sigma$-injective, if $s p(\psi)$ is $\sigma$-dense in $X$. It follows from 1.12 in [Ln11] that, for any $\varepsilon>0$ and $\mathcal{F}$, when $\delta$ is sufficiently small and $\mathcal{G}$ is sufficiently large, $\Sigma_{\varepsilon}(\psi, \mathcal{F})$ is not empty.

Definition 0.3. Let $B$ be a $C^{*}$-algebra and $X$ be a compact metric space. A homomorphism $\psi: C(X) \rightarrow B$ has finite dimensional range if (and only if) there exist a finite subset $\{\xi\}_{i=1}^{l} \subset X$ and a finite subset of mutually orthogonal projections $\left\{p_{i}\right\}_{i=1}^{l} \subset B$ such that

$$
\psi(f)=\sum_{i=1}^{l} f\left(\xi_{i}\right) p_{i} \quad \text { for all } f \in C(X) .
$$

Definition 0.4. Let $X$ be a finite CW complex and let $A$ be a unital $C^{*}$-algebra. Suppose that $\phi: C(X) \rightarrow A \otimes \mathcal{K}$ is a homomorphism and $\xi_{1}, \xi_{2}, \ldots, \xi_{m} \in X$ are points in each connected component of $X$. Let $Y=X \backslash\left\{\xi_{1}, \ldots, \xi_{m}\right\}$. Homomorphism $\phi$ gives a homomorphism $\phi_{0}: C_{0}(Y) \rightarrow A \otimes \mathcal{K}$. Let $[\phi]$ be the element in $K K(C(X), A)$ and let $\left[\phi_{0}\right]$ be an element in $K K\left(C_{0}(Y), A\right)$. We denote by $\mathcal{N}^{\prime}(X, A)$ (or just $\mathcal{N}^{\prime}$ if $X$ and $A$ are understood) the set of those elements in $K K(C(X), A)$ which are represented by those $\phi$ such that $\left[\phi_{0}\right]=0$. Given $m$ mutually orthogonal projections $p_{1}, p_{2}, \ldots, p_{m} \in A \otimes \mathcal{K}$, define $\phi^{\prime}(f)=\sum_{i=1}^{m} f\left(\xi_{i}\right) p_{i}$ for $f \in C(X)$. Then $\left[\phi^{\prime}\right] \in \mathcal{N}^{\prime}$. Conversely, if $[\phi] \in \mathcal{N}^{\prime}$, let $f_{1}, f_{2}, \ldots, f_{m}$ be projections in $C(X)$ corresponding to each component of $X$, and let $\phi\left(f_{i}\right)=p_{i}, i=1,2, \ldots, m$. Then $[\phi]-\left[\phi^{\prime}\right]=0$ in $K K(C(X), A)$. In fact, from the six-term exact sequence in $K K$ theory, the map from $K K(C(X), A)$ to $K K\left(C_{0}(Y), A\right)$ maps both $[\phi]$ and $\left[\phi^{\prime}\right]$ into zero. So they both are in the image of the map from $K K\left(C(X) / C_{0}(Y), A\right)$ (to $K K(C(X), A)$ ). Note that $C(X) / C_{0}(Y)$ is $m$ copies of $\mathbf{C}$ corresponding to the $m$ components. From the choice of $\phi^{\prime}$, they both induce the same element in $K K(C(X), A)$.

Now let $X$ be any compact metric space. Then $C(X)=\lim _{n \rightarrow \infty} C\left(X_{n}\right)$, where $X_{n}$ is a finite $\mathrm{CW}$ complex. There is a surjective map $s: K K(C(X), A)$ $\rightarrow \lim _{n \rightarrow \infty} K K\left(C\left(X_{n}\right), A\right)$. We denote by $\mathcal{N}^{\prime}$ the set of those elements $x$ in $K K(C(X), A)$ such that $s(x) \in \lim _{n \rightarrow \infty} \mathcal{N}^{\prime}\left(X_{n}, A\right)$ for any sequence of finite CW complexes $\left\{X_{n}\right\}$.

Recall that $K L(C(X), A)$ is the quotient $K K(C(X), A)$ by the subgroup of pure extensions in $\operatorname{Ext}\left(K_{*}(C(X)), K_{*-1}(A)\right)$ (see $\left.[\operatorname{Rr} 2]\right)$.

We denote by $\mathcal{N}$ the image of $\mathcal{N}^{\prime}$ in $K L(C(X), A)$. If $\phi: C(X) \rightarrow A$ is a homomorphism, we write $\Gamma(\phi) \in \mathcal{N}$, if $[\phi] \in \mathcal{N}$.

Definition 0.5. The standard definition of mod- $p K$ theory for $C^{*}$-algebras as given by Schochet in $[\mathrm{Sch}]$ is

$$
K_{i}(A ; \mathbf{Z} / n)=K_{i}\left(A \otimes C_{0}\left(X_{n}\right)\right),
$$

where $X_{n}=C_{n} \backslash\{\xi\}, \xi$ is a base point of $C_{n}$ and $C_{n}$ is the 2-dimensional CWcomplex obtained by attaching a 2-cell to $S^{1}$ via the degree $n$ map from $S^{1}$ to $S^{1}$, (notice that $K_{0}\left(C_{0}\left(X_{n}\right)\right)=\mathbf{Z} / n \mathbf{Z}$ and $K_{1}\left(C_{0}\left(X_{n}\right)\right)=\{0\}$ ). Let $A$ be a $C^{*}$-algebra. Following [DL3], we write

$$
\underline{K}(A)=\bigoplus_{i=0,1, n \geq 0} K_{i}(A ; \mathbf{Z} / n \mathbf{Z}) .
$$


By [DL3], there is an isomorphism from $K L(C(X), A)$ onto $\operatorname{Hom}_{\Lambda}(\underline{K}(C(X)), \underline{K}(A))$. Note that

$$
K_{0}\left(A \otimes C_{0}\left(C_{m} \times S^{1}\right)\right) \cong K_{0}(A) \oplus K_{1}(A) \oplus K_{0}(A ; \mathbf{Z} / m) \oplus K_{1}(A ; \mathbf{Z} / m) .
$$

There is an obvious surjective from $\bigcup_{m>0} K_{0}\left(A \otimes C\left(C_{m} \times S^{1}\right)\right)$ onto $\underline{K}(A)$. (Please see [DL3] for more information.)

The reason that we introduce mod- $p K$-theory is to deal with the case when $K_{i}(C(X))$ has torsion, $i=0,1$. If the reader is only interested in the case when $K_{i}(C(X))$ is torsion free, then it will be enough to consider $K_{0}(C(X)) \oplus$ $K_{0}\left(C(X) \otimes C\left(S^{1}\right)\right)$. Please see Remark 1.13.

0.6. Let $A$ be a $C^{*}$-algebra. Denote by $\mathbf{P}(A)$ the set of projections in

$$
\bigcup_{m \geq 0} M_{\infty}\left(A \otimes C\left(C_{m} \times S^{1}\right)\right) .
$$

Let $\mathcal{P}$ be a finite subset in $\mathbf{P}(A)$. There are a finite subset $\mathcal{G}(\mathcal{P}) \subset A$ and $\delta(\mathcal{P})>0$ such that if $B$ is any $C^{*}$-algebra and $\phi: A \rightarrow B$ is a *-preserving linear map which is $\delta(\mathcal{P})-\mathcal{G}(\mathcal{P})$-multiplicative, then

$$
\left\|((\phi \otimes i d)(p))^{2}-(\phi \otimes i d)(p)\right\|<1 / 4
$$

for all $p \in \mathcal{P}$. Hence, for each $p \in \mathcal{P}$, there is a projection $q \in \mathbf{P}(B)$ such that

$$
\|(\phi \otimes i d)(p)-q\|<1 / 2 .
$$

Furthermore, if $q^{\prime}$ is another projection satisfying the same condition, then $\| q-$ $q^{\prime} \|<1$, hence $q$ is unitarily equivalent to $q^{\prime}$. Let $\overline{\mathcal{P}}$ be the image of $\mathcal{P}$ in $\underline{K}(A)$. For each $p \in \mathcal{P}$, we set $\phi_{*}([p])=[q]$. This defines a map $\phi_{*}: \overline{\mathcal{P}} \rightarrow \underline{K}(B)$.

Let $\alpha: \overline{\mathcal{P}} \rightarrow \underline{K}(B)$. Suppose that there is a homomorphism $\psi: C(X) \rightarrow M_{k}(B)$ for some integer $k$ with finite dimensional range such that $\psi_{*}=\alpha: \overline{\mathcal{P}} \rightarrow \underline{K}(B)$. Then we write $\alpha(\overline{\mathcal{P}}) \in \mathcal{N}$.

\section{Approximation By homomorphisms With Finite DimENSIONAL RANGE}

1.1. Recall (3.1 in [FR]) that a simple $C^{*}$-algebra $A$ has property (IR) if the set of invertible elements is dense in the set of those elements in $A$ which are either invertible or not one-sided invertible. We have the following fact:

Proposition 1.2. For a simple $C^{*}$-algebra $A$, the following are equivalent:

(1) A has the property (IR);

(2) A is extremally rich;

(3) A is either of stable rank one or $A$ is purely infinite.

Proof. Suppose that $A$ is a simple $C^{*}$-algebra which has (IR). Let $x \in A$ which is not quasi-invertible (see p. 118 in [BP2]). Then, since $A$ is simple, $x$ is neither invertible nor one-sided invertible. But $A$ has (IR). So $x$ is approximated by invertible elements. Therefore the set of quasi-invertible elements is dense in $A$. So $A$ is extremally rich (section 3 in [BP2]).

Now suppose that $A$ is a simple $C^{*}$-algebra which is extremally rich. It follows from 10.5 in [BP3] that $A$ is either purely infinite simple or has stable rank one.

Suppose that $A$ is either purely infinite simple or has stable rank one. Then, by 3.1 in $[\mathrm{FR}], A$ has property (IR). 
1.3. Let $\mathbf{B}$ denote the family of those unital simple $C^{*}$-algebras with real rank zero which satisfy one of the conditions in Proposition 1.2.

Theorem $1.4([\operatorname{Ln} 12])$. Let $A \in \mathbf{B}$. Then, for any $\varepsilon>0$, if $x$ is a normal element in $A$ and

$$
\lambda-x \in \operatorname{Inv}_{0}(A)
$$

for all $\lambda \notin\{t: \operatorname{dist}(\lambda, s p(x)) \geq r\}$ for some $0 \leq r<\varepsilon$, then there is a normal element $y \in A$ with finite spectrum such that

$$
\|x-y\|<\varepsilon .
$$

We note that the condition that $A$ is simple is necessary (see [Ln12]).

Theorem $1.5([\mathrm{FR}])$. Let $D$ be the unit disk in the plane. For any $\varepsilon>0$ and any finite subset of the plane $\mathcal{F} \subset C(D)$, there exist $\delta>0$ and a finite subset $\mathcal{G} \subset C(D)$ satisfying the following: if $\Lambda: C(D) \rightarrow A$ is a contractive positive linear morphism which is $\delta$-G-multiplicative, then there exists a homomorphism $h: C(D) \rightarrow A$ such that

$$
\|\Lambda(f)-h(f)\|<\varepsilon
$$

for all $f \in \mathcal{F}$, whenever $A$ is a $C^{*}$-algebra with (IR).

Suppose that $u$ is a unitary in a unital $C^{*}$-algebra $A$ and $\Lambda: A \rightarrow B$ is a $\delta$ - $\mathcal{G}$-multiplicative contractive positive linear morphism, where $B$ is also a unital $C^{*}$-algebra. It is easy to see that $\Lambda(u)$ is close to a unitary in $B$, if $\delta$ is small enough and $\mathcal{G}$ is large enough. Since two unitaries with distance less than 1 are connected to each other by a continuous path, $\Lambda(u)$ defines an element in $U(A)$, the unitary group of $A$. We will denote it by $\Lambda_{*}([u])$. Let $\mathcal{U}$ be a finite subset of $U(A)$. There is a finite subset $\mathcal{G}(\mathcal{U}) \subset A$ and $\delta(\mathcal{U})>0$ such that if $\Lambda: A \rightarrow B$ is a $\delta(\mathcal{U})-\mathcal{G}(\mathcal{U})$-multiplicative contractive positive linear morphism, then $\Lambda_{*}([u])$ is well defined for all $u \in \mathcal{U}$.

Theorem 1.6. Let $X$ be a compact subset of the plane. For any $\varepsilon>0$ and a finite subset $\mathcal{F} \subset C(X)$, there are a finite subset $\mathcal{U}$ of the generators of $U(C(X))$, the unitary group of $C(X)$, a positive number $\delta(<\delta(\mathcal{U}))$, a finite subset $\mathcal{G} \subset C(X)$ containing $\mathcal{F}$ and $1>\sigma>0$ satisfying the following: if $A \in \mathbf{B}$ and $\Lambda: C(X) \rightarrow A$ is a contractive positive linear morphism which is $\delta$-G-multiplicative and $\sigma-\mathcal{F}$-injective with

$$
\Lambda_{*}([u])=0 \text { for all } u \in \mathcal{U},
$$

then there exists a homomorphism $h: C(X) \rightarrow A$ with finite dimensional range such that

$$
\|\Lambda(f)-h(f)\|<\varepsilon
$$

for all $f \in \mathcal{F}$.

Proof. Without loss of generality, we may assume that $X \subset D$. Let $s: C(D) \rightarrow$ $C(X)$ be the surjective map. Assume given a contractive positive linear morphism $\Lambda: C(X) \rightarrow A$. Let $\psi=\Lambda \circ s$. By applying Theorem 1.5, for any $\varepsilon_{1}>0$ with sufficiently small $\delta$ and sufficiently large $\mathcal{G}$, there is a homomorphism $h_{1}: C(D) \rightarrow$ $A$ such that

$$
\left\|\psi(f)-h_{1}(f)\right\|<\varepsilon_{1}
$$


for all $f \in \mathcal{F}_{1}$, where $\mathcal{F}_{1}$ is a finite subset of $C(D)$ such that $s\left(\mathcal{F}_{1}\right)=\mathcal{F}$, provided that $\Lambda$ is $\delta$-G-multiplicative. Let $\sigma>0$. Suppose that $\Lambda$ is also $\sigma$-injective. With small $\varepsilon_{1}$ and a large $\mathcal{G}$, we may further assume that

$$
\sup \left\{\operatorname{dist}\left(\xi, s p\left(h_{1}\right)\right), \operatorname{dist}(X, \zeta): \xi \in X, \zeta \in \operatorname{sp}\left(h_{1}\right)\right\}<2 \sigma .
$$

Let $\Omega=\{\xi: \operatorname{dist}(\xi, X) \leq 2 \sigma\}$. Note that $\Omega$ is homeomorphic to a finite CW complex on the plane. Let $x$ be the generator of $h_{1}(C(D))$ with $s p(x)=s p\left(h_{1}\right)$. It is easy to compute that, with a sufficiently large $\mathcal{U}$, the condition that $\Lambda_{*}([u])=0$ for all $u \in \mathcal{U}$ implies that $\lambda-x \in \operatorname{Inv}_{0}(A)$ for all $\lambda \in \Omega$. So, by Theorem 1.4, with sufficiently small $\sigma$ and $\varepsilon_{1}$ and with sufficiently large $\mathcal{G}$, there is a homomorphism $h: C(X) \rightarrow A$ with finite dimensional range such that

$$
\left\|h_{1}(f)-h \circ s(f)\right\|<\varepsilon / 2
$$

for all $f \in \mathcal{F}_{1}$. From this, we conclude that

$$
\|\Lambda(f)-h(f)\|<\varepsilon
$$

for all $f \in \mathcal{F}$, if $\delta$ is sufficiently small and $\mathcal{G}$ is sufficiently large.

Remark 1.7. In Theorem 1.6, if we assume that $X \subset D, \sigma$ depends on $\varepsilon$ and $\mathcal{F}$ only. Any $\sigma>0$ that satisfies

$$
\left|f(t)-f\left(t^{\prime}\right)\right|<\varepsilon / 2,
$$

whenever $\operatorname{dist}\left(t, t^{\prime}\right)<2 \sigma$ and $f \in \mathcal{F}$, will do.

For future use, given an $\varepsilon>0$, a compact metric space $X$ and a finite subset $\mathcal{F} \subset C(X)$, let $\sigma=\sigma_{X, \mathcal{F}, \varepsilon}$ be the largest positive number such that

$$
\left|f(\xi)-f\left(\xi^{\prime}\right)\right|<\varepsilon
$$

whenever $\operatorname{dist}\left(\xi, \xi^{\prime}\right)<\sigma$ and $f \in \mathcal{F}$. Note that

$$
\sigma_{F, s(\mathcal{F}), \varepsilon} \geq \sigma_{X, \mathcal{F}, \varepsilon}
$$

if $F \subset X$ is a compact subset and $s: C(X) \rightarrow C(F)$ is the surjective map. We also note that, in 1.6, the assumption that $A$ is simple is necessary since it is necessary in Theorem 1.4.

Lemma 1.8. Let $X$ be a compact metric space, $F \subset X$ be a compact subset of $X$ which is homeomorphic to a contractible compact subset of the plane and $F_{0}$ be a compact subset of $F$ with $\operatorname{dist}\left(F_{0}, X \backslash F\right)>0$. For any $\varepsilon>0$ and any finite subset $\mathcal{F} \subset C(X)$, there exist $\delta>0, \sigma>0, \eta>0$ and a finite subset $\mathcal{G} \subset C(X)$ (containing $\mathcal{F}$ ) satisfying the following: for any $C^{*}$-algebra $A \in \mathbf{B}$ and two $m u$ tually orthogonal projections $p, q \in A$, any contractive positive linear morphism $\Lambda: C(X) \rightarrow p A p$ which is $\delta$-G-multiplicative and $\sigma$-injective, and any homomorphism $h_{0}: C\left(F_{0}\right) \rightarrow q A q$, if there is a homomorphism $h_{1}: C(X) \rightarrow(p+q) A(p+q)$ with finite dimensional range such that

$$
\left\|\Lambda(f) \oplus h_{0} \circ s(f)-h_{1}(f)\right\|<\eta
$$

for all $f \in \mathcal{G}$, where $s: C(X) \rightarrow C\left(F_{0}\right)$ is the surjective map, then there is a homomorphism $h: C(X) \rightarrow p A p$ with finite dimensional range such that

$$
\|\Lambda(f)-h(f)\|<\varepsilon
$$

for all $f \in \mathcal{F}$. 
Proof. We may assume that $p+q=1$ and $\mathcal{F}$ is a subset of the unit ball of $C(X)$.

It follows from 1.6 that, for any $\varepsilon_{1}>0$ and a finite subset $\mathcal{F}_{1} \in C(F)$, there are $\delta_{1}>0, \sigma_{1}>0$ and a finite subset $\mathcal{G}_{1} \in C(F)$ satisfying the following: if $\lambda: C(F) \rightarrow$ $B$ is a $\delta_{1}-\mathcal{G}_{1}$-multiplicative and $\sigma_{1}$-injective contractive positive linear morphism, then there exists a homomorphism $H_{1}: C(F) \rightarrow B$ with finite dimensional range such that

$$
\left\|\lambda(f)-H_{1}(f)\right\|<\varepsilon_{1}
$$

for all $f \in \mathcal{F}_{1}$, whenever $B \in \mathbf{B}$.

Let $s_{1}: C(X) \rightarrow C(F)$ be the surjective map. Let $f_{1}, f_{2} \in C(X)$ such that $0 \leq f_{1} \leq 1, f_{1}(x)=0$ if $x \in F_{0}, f_{1}(x)=1$ if $x \in X \backslash F$, and $0 \leq f_{1} \leq 1, f_{2}(x)=1$ if $x \in F_{0}, f_{2}(x)=0$ if $x \in X \backslash F$ and $f_{1} f_{2}=0$.

Let $\mathcal{G}_{2} \subset C(X)$ be a finite subset containing $f_{1}, f_{2}$ and containing a finite subset of $C(X)$ such that its image under $s_{1}$ contains $\mathcal{G}_{1}$. Let $\delta>0, \eta>0$ and $\sigma>0$. Suppose that $\Lambda, h_{0}$ and $h_{1}$ are given as stated in the lemma for above $\delta, \sigma$ and $\mathcal{G}$. Note that we may assume that $\mathcal{G}$ is a subset of the unit ball of $C(X)$.

Write $h_{1}=\sum_{i=1}^{l} f\left(\xi_{i}\right) p_{i}$ for all $f \in C(X)$, where $\left\{x_{i}\right\}$ are fixed points in $X$ and $\left\{p_{i}\right\}$ are finitely many mutually orthogonal projections in $A$. Set $\phi_{1}(f)=$ $\sum_{\xi \in X \backslash F} f\left(\xi_{i}\right) p_{i}, \phi_{2}(f)=\sum_{\xi \in F} f\left(\xi_{i}\right) p_{i}$ for all $f \in C(X)$ and let $r=\sum_{\xi_{i} \in X \backslash F} p_{i}$. Since $r$ commutes with $h_{1}$ and $q$ commutes with $\Lambda \oplus h_{0} \circ s$, we have

$$
h_{1}\left(f_{1}\right) r=r h_{1}\left(f_{1}\right)=r
$$

and

$$
\left(\Lambda \oplus h_{0} \circ s\right)\left(f_{2}\right) q=q\left(\left(\Lambda \oplus h_{0} \circ s\right)\left(f_{2}\right)\right)=q .
$$

Therefore

$$
\begin{aligned}
\|r q\| & =\left\|r h_{1}\left(f_{1}\right)\left(\Lambda \oplus h_{0} \circ s\right)\left(f_{2}\right) q\right\| \\
& \leq\left\|r\left(h_{1}\left(f_{1}\right)-\left(\Lambda\left(f_{1}\right) \oplus h_{0} \circ s\left(f_{1}\right)\right)\right)\right\|+\left\|r\left(\Lambda \oplus h_{0} \circ s\right)\left(f_{1}\right)\left(\Lambda \oplus h_{0} \circ s\right)\left(f_{2}\right) q\right\| \\
& <\eta+\delta .
\end{aligned}
$$

Consequently,

$$
\|r-\operatorname{prp}\|<2(\eta+\delta)
$$

If $\delta+\eta<1 / 4$, by [Eff, A8], there is a projection $r^{\prime} \leq p$ such that

$$
\left\|r^{\prime}-r\right\|<2(\eta+\delta)
$$

and there is a unitary $u_{1} \in(p+q) A(p+q)$ such that

$$
\left\|u_{1}-(p+q)\right\|<4(\eta+\delta) \text { and } u_{1}^{*} r u_{1}=r^{\prime} .
$$

Thus

$$
\left\|u_{1}^{*} \phi_{i}(f) u_{1}-\phi_{i}(f)\right\|<8(\eta+\delta)\|f\|, \quad i=1,2,
$$

for all $f \in C(X)$. Then

$$
\begin{gathered}
\left\|p \phi_{2}(f)-\phi_{2}(f) p\right\| \leq\left\|p \phi_{1}(f)-\phi_{1}(f) p\right\|+\left\|p h_{1}(f)-h_{1}(f) p\right\| \\
\leq\left\|p r \phi_{1}(f)-\phi_{1}(f) r p\right\|+2(\eta+\delta)<4(\eta+\delta)
\end{gathered}
$$

for all $f \in \mathcal{G}$. Since

$$
\left\|u_{1}^{*} \phi_{2}(f) u_{1}-\phi_{2}(f)\right\|<8(\eta+\delta)\|f\|
$$


for all $f \in C(X)$, we obtain

$$
\left\|p\left(u_{1}^{*} \phi_{2}(f) u_{1}\right)-\left(u_{1}^{*} \phi_{2}(f) u_{1}\right) p\right\| \leq 4(\eta+\delta)+16(\eta+\delta)=20(\eta+\delta)\|f\|
$$

for all $f \in \mathcal{G}$. Put $p^{\prime}=p-r^{\prime}$. Since

$$
r^{\prime} u_{1}^{*} \phi_{2}(f) u_{1}=u_{1}^{*} \phi_{2}(f) u_{1} r^{\prime}=0
$$

for all $f \in C(X)$,

$$
\left\|p^{\prime}\left(u_{1}^{*} \phi_{2}(f) u_{1}\right)-\left(u_{1}^{*} \phi_{2}(f) u_{1}\right) p^{\prime}\right\|<22(\eta+\delta)
$$

for all $f \in \mathcal{G}$. Define $L(f)=p^{\prime}\left(u_{1}^{*} \phi_{2} \circ s_{1}(f) u_{1}\right) p^{\prime}$ for all $f \in C(F)$. We have

$$
\left\|p h(f) p-u_{1}^{*} \phi_{1}(f) u_{1} \oplus L(f)\right\|<8(\eta+\delta)
$$

for all $f \in \mathcal{G}$.

It is clear that if $\sigma, \delta$ and $\eta$ are small enough and $\mathcal{G}$ is large enough, $\left\{\xi_{i}: \xi_{i} \in F\right\}$ is $\sigma_{1}$-dense in $F$. So, when $\sigma, \delta$ and $\eta$ are small enough and $\mathcal{G}$ is large enough, $L$ is a $\delta_{1}$ $\mathcal{G}_{1}$-multiplicative and $\sigma_{1}$-injective contractive positive linear morphism from $C(F)$ into $p^{\prime} A p^{\prime}$. By applying Lemma 1.4, there is a homomorphism $H_{2}: C(F) \rightarrow p^{\prime} A p^{\prime}$ with finite dimensional range such that

$$
\left\|L(f)-H_{2}(f)\right\|<\varepsilon_{1}
$$

for all $f \in s(\mathcal{F})$.

Since $\Lambda(f)=p\left(\Lambda \oplus h_{0} \circ s\right) p$, we have

$$
\left\|\Lambda(f)-u_{1}^{*} \phi_{1}(f) u_{1} \oplus H_{2} \circ s(f)\right\|<\varepsilon
$$

for all $f \in \mathcal{F}$, provided that $\varepsilon_{1}, \delta$ and $\eta$ are small enough.

Lemma 1.9. Let $X$ be a compact metric space and let $F_{0} \subset X$ be a compact subset of $X$ which is homeomorphic to a contractible compact subset of the plane. Suppose that there is $d>0$ such that there is a retraction $r: X_{d} \rightarrow F_{0}$, where $X_{d}=\left\{\xi \in X: \operatorname{dist}\left(x, F_{0}\right) \leq d\right\}$. For any $\varepsilon>0$ and a finite subset $\mathcal{F} \subset C(X)$, there exist $\delta>0, \eta>0$ and a finite subset $\mathcal{G} \subset C(X)$ (containing $\mathcal{F}$ ) satisfying the following: For any $C^{*}$-algebra $A \in \mathbf{B}$ and two mutually orthogonal projections $p, q \in A$, any contractive positive linear morphism $\Lambda: C(X) \rightarrow p A p$ which is $\delta$ $\mathcal{G}$-multiplicative and $\sigma_{X, \mathcal{F}, \varepsilon}$-injective, and any homomorphism $h_{0}: C\left(F_{0}\right) \rightarrow q A q$, if there is a homomorphism $h_{1}: C(X) \rightarrow(p+q) A(p+q)$ with finite dimensional range such that

$$
\left\|\Lambda(f) \oplus h_{0} \circ s(f)-h_{1}(f)\right\|<\eta
$$

for all $f \in \mathcal{G}$, where $s: C(X) \rightarrow C\left(F_{0}\right)$ is the canonical surjective map, then there is a homomorphism $h: C(X) \rightarrow p A p$ with finite dimensional range such that

$$
\|\Lambda(f)-h(f)\|<\varepsilon
$$

for all $f \in \mathcal{F}$.

Proof. The proof is a minor modification of that of Lemma 1.8. Since $F_{0}$ is compact, there is $0<a<d$ such that

$$
\left\|f \circ r^{\prime}-f\right\|<\varepsilon / 2
$$

for all $f \in s(\mathcal{F})$, where $r^{\prime}=\left.r\right|_{X_{a}}, X_{a}=\left\{\xi \in X_{d}: \operatorname{dist}\left(\xi, F_{0}\right) \leq a\right\}$ and $s: C(X) \rightarrow$ $C\left(X_{d}\right)$. 
In the proof of Lemma 1.8, we let $F=X_{a}$. Let $j: C\left(F_{0}\right) \rightarrow C(F)$ by defining $j(f)=f \circ r^{\prime}$ for $f \in C\left(F_{0}\right)\left(F=X_{a}\right)$ and $s^{\prime}: C(F) \rightarrow C\left(F_{0}\right)$ be the surjective map. Then

$$
\left\|j \circ s^{\prime}(f)-f\right\|<\varepsilon / 2
$$

for all $f \in s(\mathcal{F})$. Define $L^{\prime}=L \circ j$, where $L$ is as defined in the proof of Lemma 1.8. Now $L: C\left(F_{0}\right) \rightarrow p^{\prime} A p^{\prime}$ is a homomorphism and $F_{0}$ is homeomorphic to a contractive subset of the plane. We then apply Lemma 1.4 to $L$ as in the proof of Lemma 1.8 to obtain a homomorphism $H_{2}: C\left(F_{0}\right) \rightarrow p^{\prime} A p^{\prime}$ such that

$$
\left\|L^{\prime}(f)-H_{2}(f)\right\|<\varepsilon_{1}
$$

for all $f \in s^{\prime} \circ s(\mathcal{F})$. We note that

$$
\left\|L(f)-L^{\prime} \circ s^{\prime}(f)\right\|<\varepsilon / 2
$$

for all $f \in s(\mathcal{F})$. Thus, as in the proof of Lemma 1.8, we will have

$$
\left\|\Lambda(f)-u_{1}^{*} \phi_{1}(f) U_{1} \oplus H_{2} \circ s^{\prime} \circ s(f)\right\|<\varepsilon
$$

for all $f \in \mathcal{F}$, provided that $\varepsilon_{1}, \delta$ and $\eta$ are small enough.

Corollary 1.10. Let $X$ be a compact metric space and let $F \subset X$ be a compact subset of $X$ which is a finite $C W$ complex of dimension no more than 1. Suppose that there is $d>0$ such that there is a retraction $r: X_{d} \rightarrow F$, where $X_{d}=\{\xi \in$ $X: \operatorname{dist}(x, F) \leq d\}$. For any $\varepsilon>0$ and a finite subset $\mathcal{F} \subset C(X)$, there exist $\delta>0, \sigma>0, \eta>0$ and a finite subset $\mathcal{G} \subset C(X)$ (containing $\mathcal{F}$ ) satisfying the following: for any $C^{*}$-algebra $A \in \mathbf{B}$ and two mutually orthogonal projections $p, q \in A$, any contractive positive linear morphism $\Lambda: C(X) \rightarrow p A p$ which is $\delta$ $\mathcal{G}$-multiplicative and $\sigma$-injective, and any homomorphism $h_{0}: C(F) \rightarrow q A q$ with finite dimensional range, if there is a homomorphism $h_{1}: C(X) \rightarrow(p+q) A(p+q)$ with finite dimensional range such that

$$
\left\|\Lambda(f) \oplus h_{0} \circ s(f)-h_{1}(f)\right\|<\eta
$$

for all $f \in \mathcal{G}$, where $s: C(X) \rightarrow C(F)$ is the surjective map, then there is a homomorphism $h: C(X) \rightarrow p A p$ with finite dimensional range such that

$$
\|\Lambda(f)-h(f)\|<\varepsilon
$$

for all $f \in \mathcal{F}$.

Proof. We note that $F$ is homeomorphic to $F^{\prime}=\bigcup_{j=1}^{m} X_{i}$, where each $X_{i}$ is a point or a closed line segment, and these line segments only intersect at the end points and do not intersect with anything else. Note that each line segment is a compact contractive subset of the plane. Let $Y_{i}$ be the compact subset of $F$ which is homeomorphic to $X_{i}$. It is clear that, for each $i$, there is a retraction $r_{i}: X_{d}^{(i)} \rightarrow Y_{i}$, where $X_{d}^{(i)}=\left\{x \in X: \operatorname{dist}\left(x, Y_{i}\right) \leq d\right\}$, since there is a retraction $r: X_{d} \rightarrow F$. Thus the corollary follows by a repeated ( $m$ times) application of Lemma 1.8.

Lemma 1.11. Let $X$ be a compact metric space with dimension no more than 2 and let $\mathcal{F}$ be a finite subset of (the unit ball of) $C(X)$. For any $\varepsilon>0$, there exist a finite subset $\mathcal{P}$ of projections in $\mathbf{P}(C(X))$, positive numbers $\delta>0(\delta<\delta(\mathcal{P})$ in $0.6)$ and $\sigma>0$ and a finite subset $\mathcal{G}$ of (the unit ball of) $C(X)$ such that whenever $A \in \mathbf{B}$ and whenever $\psi: C(X) \rightarrow A$ is a contractive unital positive linear map 
which is $\delta$-G-multiplicative, $\sigma-\mathcal{G}$-injective and $\psi_{*}(\overline{\mathcal{P}}) \in \mathcal{N}$, then there exists a unital homomorphism $\varphi: C(X) \rightarrow A$ with finite dimensional range such that

$$
\|\psi(f)-\varphi(f)\|<\varepsilon
$$

for all $f \in \mathcal{F}$.

Proof. First, we will reduce the general case to the case when $X$ is a polyhedron. We can write $C(X)=\lim _{n \rightarrow \infty}\left(C\left(X_{n}\right), f_{n, n+1}\right)$, where $f_{n, n+1}$ is the homomorphism from $C\left(X_{n}\right)$ to $C\left(X_{n+1}\right)$ and by $X_{n}$ is a polyhedron of dimension no more than 2 . We also denote by $f_{n}$ the homomorphism from $C\left(X_{n}\right)$ into $C(X)$ and by $f_{n, m}$ : $C\left(X_{n}\right) \rightarrow C\left(X_{m}\right)(m>n)$ the holomorphism induced by the direct limit system. It is easy to see that we may assume that all $f_{n}$ are surjective. In fact we may assume that $X \subset X_{n+1} \subset X_{n}$ for all $n$ and $X=\bigcap_{n=1} X_{n}$. We may also assume that there exists a finite subset $\mathcal{F}_{1} \subset C\left(X_{1}\right)$ such that $f_{1}\left(\mathcal{F}_{1}\right)=\mathcal{F}$. Since $X=\bigcap_{n=1} X_{n}$ and $X_{n+1} \subset X_{n}$, there is, for any $\varepsilon>0$, an integer $N$ such that for any $\xi \in X_{N}$, there exists a point $\zeta \in X$ such that

$$
|f(\xi)-f(\zeta)|<\varepsilon
$$

for all $f \in \mathcal{F}_{1}$. It follows from Lemma 1.1 in $[\mathrm{M}]$ that, for any $r>0$, there exist a polyhedron $Q$ of dimension no more than 2, an injective homomorphism $\tau_{1}$ : $C(Q) \rightarrow C(X)$ and a homomorphism $\tau_{2}: C\left(X_{N}\right) \rightarrow C(Q)$ such that

$$
\left\|f_{N}(g)-\tau_{1} \circ \tau_{2}(g)\right\|<r
$$

for all $g \in \mathcal{F}_{1}$. Set $\mathcal{F}_{2}=\tau_{2}\left(\mathcal{F}_{1}\right)$. Note that $\tau_{1}$ gives a homomorphism from $K L(C(X), A)$ into $K L(C(Q), A)$ and that homomorphism maps $\mathcal{N}$ in $K L(C(X), A)$ to $\mathcal{N}$ in $K L(C(Q), A)$. In particular, if a finite subset $\mathcal{P}_{1} \subset \mathbf{P}(C(Q))$ is given, then there is a finite subset $\mathcal{P} \subset \mathbf{P}(C(X))$ such that

$$
\left(\psi \circ \tau_{1}\right)_{*}\left(\overline{\mathcal{P}}_{1}\right) \in \mathcal{N} \quad(\text { see } \S 0.6 \text { for the notation })
$$

whenever

$$
\psi_{*}(\overline{\mathcal{P}}) \in \mathcal{N} .
$$

We also note that $\psi \circ \tau_{1}$ is $\sigma$ - $\mathcal{F}_{2}$-injective. Furthermore, we note that a homomorphism $\psi^{\prime}: C\left(X_{N}\right) \rightarrow A$ with finite dimensional range is close (within $\varepsilon$ on $f_{1, N}(\mathcal{F})$ ) to a homomorphism $\psi: C(X) \rightarrow A$ with finite dimensional range on $\mathcal{F}=f_{1}\left(\mathcal{F}_{1}\right)$. Thus, without loss of generality, we may assume that $X$ is a polyhedron of dimension no more than 2.

We decompose $X$ and write $X=F \cup Y$, where $F$ is finite CW complex with dimension 1 and $Y$ is finitely $(m)$ many disjoint subsets each of which is homeomorphic to the open unit disk. Let $d>0$ and $F_{d}=\{\xi \in X: \operatorname{dist}(\xi, F) \leq d\}$. Choose a small $d>0$ so that the closure $Y_{d}$ of $X \backslash F_{d}$ is a disjoint union of finitely many compact subsets $\left\{Y_{j}\right\}_{j=1}^{m}$, where each $Y_{j}$ is homeomorphic to the (closed) unit disk on the plane.

Since $\psi_{*}(\mathcal{P}) \in \mathcal{N}$ and $A$ is either purely infinite or has stable rank one, it follows from Theorem 1.6 in [Ln11] that, for any $\eta>0$ and any finite subset $\mathcal{G}_{1} \subset C(X)$, when $\delta$ is sufficiently small, $\mathcal{P}$ and $\mathcal{G}$ are sufficiently large,

$$
\left\|\psi(f) \oplus h_{0}(f)-h_{1}(f)\right\|<\eta
$$

for all $f \in \mathcal{G}$, where $h_{0}: C(X) \rightarrow M_{L}(A)$ and $h_{1}: C(X) \rightarrow M_{L+1}(A)$ are homomorphisms with finite dimensional range. Write $h_{0}(f)=\sum_{i=1}^{l} f\left(\xi_{i}\right) p_{i}$, where $\left\{\xi_{i}\right\}$ are finitely many points in $X$ and $\left\{p_{i}\right\}$ are finitely many mutually orthogonal 
projections in $M_{L}(A)$. Without loss of generality, we may assume that $\left\{\xi_{i}\right\}$ are in the union of the interior of $F_{d}$ and the interior of $\bigcup_{j=1}^{m} Y_{j}$. Let $s_{j}: C(X) \rightarrow C\left(Y_{j}\right)$ and $s_{d}: C(X) \rightarrow C\left(F_{d}\right)$ be the surjective maps. We may write

$$
h_{0}=\bigoplus_{j=1}^{m} h_{0}^{(j)} \circ s_{j} \oplus\left(h_{d} \circ s_{d}\right)
$$

where $h_{0}^{(j)}: C\left(Y_{j}\right) \rightarrow q_{j} M_{L}(A) q_{j}$ and $h_{d}: C\left(F_{d}\right) \rightarrow q_{d} M_{L}(A) q_{d}$ are homomorphisms with finite dimensional range and $q_{d}, q_{1}, q_{2}, \ldots, q_{m}$ are mutually orthogonal projections in $M_{L}(A)$. For any $\varepsilon_{1}>0$ and any finite subset $\mathcal{G}_{1} \in C(X)$, with sufficiently small $\delta, \sigma$ and $\eta$, and sufficiently large $\mathcal{G}$, by repeatedly applying Lemma 1.8 (as in Corollary 1.10), there is a homomorphism $h_{2}: C(X) \rightarrow$ $\left(1+q_{d}\right) M_{1+L}(A)\left(1+q_{d}\right)$ with finite dimensional range such that

$$
\left\|\Lambda(f) \oplus h_{d} \circ s_{d}(f)-h_{2}(f)\right\|<\varepsilon_{1} / 2
$$

for all $f \in \mathcal{G}_{1}$.

There is a retraction $r: F_{d} \rightarrow F$. Let $j: C(F) \rightarrow C\left(F_{d}\right)$ be the injection induced by $r$ and $s_{0}: C\left(X_{d}\right) \rightarrow C(F)$ be the surjective map.

We now choose $\mathcal{G}_{1}$ and $\varepsilon_{1}$ so that we can apply Corollary 1.10 for the one (or zero) dimensional finite CW complex $F$ (and for $\varepsilon>0$ and $\mathcal{F}$ ) as below. With this $\varepsilon_{1}$ and $\mathcal{G}_{1}$, we can choose small $d>0$ at the beginning so that

$$
\left\|j \circ s_{0}\left(s_{d}(f)\right)-s_{d}(f)\right\|<\varepsilon_{1} / 2
$$

for all $f \in \mathcal{G}_{1}$. Denote $h_{d} \circ j: C(F) \rightarrow q_{d} M_{L}(A) q_{d}$ by $H$. Then we have

$$
\left\|\Lambda(f) \oplus H \circ\left(s_{0} \circ s_{d}\right)(f)-h_{2}(f)\right\|<\varepsilon_{1}
$$

for all $f \in \mathcal{G}_{1}$.

Then, by applying Corollary 1.10, we obtain a homomorphism $h: C(X) \rightarrow A$ with finite dimensional range such that

$$
\|\Lambda(f)-h(f)\|<\varepsilon
$$

for all $f \in \mathcal{F}$, provided that $\varepsilon_{1}$ is small enough and $\mathcal{G}_{1}$ is large enough.

This proves the theorem for the case when $X$ is a two-dimensional polyhedron. The above certainly also work if we replace disk by line segments. So the theorem holds for the case when $X$ is one-dimensional polyhedron. (It also follows from [Lr3] and [Ln3].) The case when $X$ is zero dimensional is trivial, since $A$ has real rank zero.

Theorem 1.12. Let $X$ be a compact metric space with dimension no more than 2 and let $\mathcal{F}$ be a finite subset of (the unit ball of) $C(X)$. For any $\varepsilon>0$, there exist a finite subset $\mathcal{P}$ of projections in $\mathbf{P}(C(X))$, a positive number $\delta>0(\delta<\delta(\mathcal{P}))$ and a finite subset $\mathcal{G}$ of (the unit ball of) $C(X)$ such that whenever $A \in \mathbf{B}$ and whenever $\psi: C(X) \rightarrow A$ is a contractive unital positive linear map which is $\delta$ - $\mathcal{G}$ multiplicative and $(1 / 2) \sigma_{X, \mathcal{F}, \varepsilon / 4^{-}} \mathcal{F}$-injective, and $\psi_{*}(\overline{\mathcal{P}}) \in \mathcal{N}$, then there exists a unital homomorphism $\varphi: C(X) \rightarrow A$ with finite dimensional range such that

$$
\|\psi(f)-\varphi(f)\|<\varepsilon
$$

for all $f \in \mathcal{F}$. 
Proof. The only difference between Lemma 1.11 and Theorem 1.12 is the requirement about $\sigma$. It follows from [GL2] that we may assume that $A$ is not elementary. Let $\sigma=1 / 2 \sigma_{X, \mathcal{F}, \varepsilon / 4}$. Suppose that $\psi: C(X) \rightarrow A$ is a contractive positive linear morphism satisfying the conditions in the theorem.

Given any $\varepsilon_{1}>0$ and $\mathcal{G}_{1}$, with sufficiently small $\delta$ and sufficiently large $\mathcal{G}$, by Lemma 1.5 in [LP1], without loss of generality, we may write that

$$
\psi(f)=\sum_{i=1}^{m} f\left(\zeta_{i}\right) p_{i} \oplus \psi_{1}(f)
$$

for all $f \in C(X)$, where $\left\{\zeta_{i}\right\}$ is $2 \sigma$-dense in $X$ and $\psi_{1}$ is a $\delta_{1}-\mathcal{G}_{1}$-multiplicative contractive positive linear morphism. Since we now assume that $A$ is not elementary and simple, there exists a nonzero projection $e \in A$ such that $e \preceq p_{i}$ for each $i$. Again, for any $\sigma_{1}>0$, since $e A e$ is nonelementary and simple, there exists a homomorphism $h_{0}: C(X) \rightarrow e A e$ which is $\sigma_{1}-\mathcal{G}_{1}$-injective. Now we apply Lemma 1.11 to the map $\psi_{1} \oplus h_{0}$ (with sufficiently small $\delta_{1}$ and sufficiently large $\mathcal{G}_{1}$ ). We obtain a homomorphism $h_{1}: C(X) \rightarrow Q M_{2}(A) Q\left(\right.$ with $\left.Q=\operatorname{diag}\left(1-\sum_{i=1}^{m} p_{i}, e\right)\right)$ with finite dimensional range such that

$$
\left\|\psi_{1}(f) \oplus h_{0}(f)-h_{1}(f)\right\|<\varepsilon / 3
$$

for all $f \in \mathcal{F}$. Since $\left\{\zeta_{i}\right\}$ is $\sigma$-dense in $X$, by changing $h_{0}$ slightly, we obtain a homomorphism $h_{2}(f)=\sum_{i=1}^{m} f\left(\zeta_{i}\right) q_{i}$, where $\left\{q_{i}\right\}$ are mutually orthogonal projections in $e A e$ such that

$$
\left\|\psi_{1}(f) \oplus h_{2}(f)-h_{1}(f)\right\|<\varepsilon / 3+\varepsilon / 4
$$

for all $f \in \mathcal{F}$. Note that $q_{i} \preceq p_{i}$ for each $i$. There is a unitary $U \in M_{2}(A)$ such that

$$
U^{*} q_{i} U \leq p_{i} \quad \text { and } \quad U^{*}\left(1-\sum_{i=1}^{m} p_{i}\right)=\left(1-\sum_{i=1}^{m} p_{i}\right) U=\left(1-\sum_{i=1}^{m} p_{i}\right) .
$$

Thus

$$
\left\|\psi(f)-\left[\sum_{i=1}^{m} f\left(\xi_{i}\right)\left(p_{i}-U^{*} q_{i} U\right) \oplus U^{*} h_{2}(f) U\right]\right\|<\varepsilon
$$

for all $f \in \mathcal{F}$.

Remark 1.13. Theorem 1.12 is in the best form in the following sense: the condition that $\operatorname{dim}(X) \leq 2$ is necessary by [GL2]. Note that a homomorphism $\phi$ can be approximated (pointwise) by homomorphisms with finite dimensional range only if $[\phi] \in \mathcal{N}$ (see 5.4 in $[\operatorname{Rr} 1]$ ). So the condition that $\psi_{*}(\overline{\mathcal{P}}) \in \mathcal{N}$ is also necessary. The conditions that $A$ is simple and has real rank zero are necessary (note that the condition that $A$ is simple is necessary in Theorem 1.4). The "injective" condition cannot be more relaxed than $\sigma_{X, \mathcal{F}, \varepsilon^{-}} \mathcal{F}$-injective otherwise as stated earlier in the introduction, we would not know which $K K$-theoretical obstacle needs to vanish. We also note that we do not know if there is any simple $C^{*}$-algebra of real rank zero which is not in $\mathbf{B}$. If $K_{i}(C(X))$ is torsion free, then $K L(C(X), A)=\operatorname{Hom}\left(K_{*}(C(X)), K_{*}(A)\right)$. So, from the proof of Theorem 1.6 in [Ln11], one sees that the condition that $\psi_{*}(\overline{\mathcal{P}}) \in \mathcal{N}$ in Lemma 1.11 and in Theorem 1.12 can be replaced by the condition that $\psi_{*}(\overline{\mathcal{Q}})=h_{*}(\overline{\mathcal{Q}})$ for some homomorphism $h: C(X) \rightarrow A \otimes \mathcal{K}$ with finite dimensional range and some (large) finite subset $\mathcal{Q}$ of projections in $\bigcup_{m} M_{m}(C(X)) \oplus M_{m}\left(C(X) \otimes C\left(S^{1}\right)\right.$. 
However, if $K_{0}(C(X))$ has torsion, then we have to use the mod- $p K$-theory since $K L(C(X), A) \neq \operatorname{Hom}\left(K_{*}(C(X)), K_{*}(A)\right)$. The following is an easy example. Let $D_{n}=C\left(C_{n}\right)$, where $C_{n}$ is as in 0.5 and $n>2$. Note that $\operatorname{dim}\left(C_{n}\right)=2$, $K_{0}\left(D_{n}\right)=\mathbf{Z} \oplus \mathbf{Z} / n \mathbf{Z}$ and $K_{1}\left(D_{n}\right)=\{0\}$. Let $A$ be a separable unital (nuclear) purely infinite simple $C^{*}$-algebra with $K_{0}(A)=0$ and $K_{1}(A)=\mathbf{Z}$. Such a $C^{*}$-algebra is given in [Rr1]. From the Universal Coefficient Theorem, since $\operatorname{Hom}\left(K_{*}\left(C\left(C_{n}\right)\right), K_{*}(A)\right)=0, K K\left(D_{n}, A\right)=\operatorname{ext}_{\mathbf{Z}}^{1}(\mathbf{Z} / n \mathbf{Z}, \mathbf{Z})$. Choose a nontrivial element $\alpha \in \operatorname{ext}_{\mathbf{Z}}^{1}(\mathbf{Z} / n \mathbf{Z}, \mathbf{Z})$. Then, by Theorem 1.17 in [Ln11], there is a unital homomorphism $h: D_{n} \rightarrow A$ such that $[h]=\alpha$. Let $F: D_{n} \rightarrow A$ be a pointevaluation. Then $[F]=0$ in $K K\left(D_{n}, A\right)$. We see that $h$ cannot be approximated by homomorphisms with finite dimensional range, since $[h] \neq[F]$ in $K K\left(D_{n}, A\right)$ (5.4 in $[\mathrm{Rr} 1])$. However,

$$
h_{*}([p])=F_{*}([p])=0
$$

for any projection in $\bigcup_{m} M_{m}\left(D_{n}\right) \oplus M_{m}\left(D_{n} \otimes C\left(S^{1}\right)\right)$. So to reveal the hidden obstacle in $\operatorname{ext}_{\mathbf{Z}}^{1}\left(K_{0}\left(D_{n}\right), K_{1}(A)\right)$, we use mod- $p K$-theory.

Corollary 1.14. Let $X$ be a compact metric space of dimension no more than two. For any $\varepsilon>0$ and any finite subset $\mathcal{F} \subset C(X)$, there is $\sigma>0$ such that, for any unital $C^{*}$-algebra $A \in \mathbf{B}$ and $\sigma$-injective homomorphism $\phi: C(X) \rightarrow A$ with

$$
\Gamma(\phi) \in \mathcal{N}
$$

there is a homomorphism $h: C(X) \rightarrow A$ with finite dimensional range such that

$$
\|\phi(f)-h(f)\|<\varepsilon
$$

for all $f \in \mathcal{F}$.

\section{NON-INJECTIVE MAPS}

While we have seen that the "injective" condition is necessary for Theorem 1.12, in some cases, however, this condition can be removed, if we do not require the homomorphism $\phi$ in Theorem 1.12 to have finite dimensional range. Furthermore, a homomorphism $\phi: C(X) \rightarrow A$ may have the property that $\Gamma(\phi) \notin \mathcal{N}$. So if we just want to have homomorphisms to approximate a $\delta$ - $\mathcal{G}$-multiplicative contractive positive linear morphism $\Lambda$, it is not necessary to have $\Lambda_{*}(\mathcal{P}) \in \mathcal{N}$. However, in general, some $K$-theoretical condition is still needed.

2.1. Let $X$ be a compact metric space and $A$ be a $C^{*}$-algebra. There is a short exact sequence

$$
\operatorname{kerd}_{X} \rightarrow K_{0}(C(X)) \rightarrow C(X, \mathbf{Z}) \rightarrow 0,
$$

where the map $d: K_{0}(C(X)) \rightarrow C(X, \mathbf{Z})$ is the dimension map. Suppose that there is a homomorphism $h: C(Y) \rightarrow C(X)$. Then the induced map $h_{*}$ maps $k e r d_{Y}$ into $\operatorname{kerd} d_{X}$.

Let $h: C(X) \rightarrow A$ be a homomorphism and $A$ be a $C^{*}$-algebra with normalized quasitraces $\tau_{a}$. Note that each $\tau_{a}$ gives a state on $K_{0}(A)$. For each quasitrace of $A$, $\tau_{a} \circ h$ gives a trace of $C(X)$. It is then clear that, if $b \in \operatorname{kerd}_{X}$, then $t(b)=0$, where $t$ is the state on $K_{0}(C(X))$ defined by $\tau_{a} \circ h$. So, in general, not every element $\alpha \in K L(C(X), A)$ is given by a homomorphism from $C(X)$ into $A$.

We now introduce the following definition. Denote by $\mathcal{N} k$ the set of those elements $\alpha$ in $K L(C(X), A)$ such that $\left.\gamma(\alpha)\right|_{\text {kerd }_{X}}=0$, where $\gamma: K L(C(X), A) \rightarrow$ $\operatorname{Hom}\left(K_{*}(C(X)), K_{*}(A)\right)$ is the usual surjective map. 
Lemma 2.2. Let $X$ be a locally compact mertric space and $G \subset X$ be an open subset. For any $\varepsilon>0$ and a finite subset $\mathcal{F} \subset C_{0}(X)$, there exist $\delta>0$, a finite subset $\mathcal{G}_{1} \subset I$, where $I=\left\{f \in C_{0}(X): f(\xi)=0, \xi \in X \subset G\right\}$, such that, if $A$ is a $C^{*}$-algebra and $\phi: C_{0}(X) \rightarrow A$ is a positive linear map with

$$
\|\phi(f)\|<\delta
$$

for all $f \in \mathcal{G}_{1}$, then there is a positive linear map $\Lambda: C_{0}(X \backslash G) \rightarrow A$, such that

$$
\|\phi(f)-\Lambda(s \circ f)\|<\varepsilon
$$

for all $f \in \mathcal{F}$, where $s: C_{0}(X) \rightarrow C_{0}(X \backslash G)$ is the surjective map.

Proof. Suppose that the lemma is false. Let $\mathcal{G}_{1}, \mathcal{G}_{2}, \ldots, \mathcal{G}_{n}, \ldots$ be a sequence of finite subsets of the unit ball of $I$ such that $\mathcal{G}_{n} \subset \mathcal{G}_{n+1}$ and the union $\bigcup_{n=1}^{\infty} \mathcal{G}_{n}$ is dense in the unit ball of $C(X)$. Then there are a positive number $\varepsilon>0$, a finite subset $\mathcal{F} \subset C(X)$, a sequence $\left\{\delta_{n}\right\}$ with $\delta_{n} \rightarrow 0$, unital $C^{*}$-algebras $B_{n}$ and contractive positive linear morphisms $\psi_{n}: C_{0}(X) \rightarrow B_{n}$ with $\left\|\psi_{n}(f)\right\|<\delta_{n}$ for $f \in \mathcal{G}_{n}$ such that

$$
\inf \left\{\sup _{f \in \mathcal{F}}\left\{\left\|\psi_{n}(f)-\Lambda_{n}(s \circ f)\right\|\right\}\right\} \geq \varepsilon .
$$

Here the infimum is taken over all contractive positive linear morphisms

$$
\Lambda_{n}: C_{0}(X \backslash G) \rightarrow A
$$

Let $\Psi=\left\{\psi_{n}\right\}$. Then $\Psi: C(X) \rightarrow \prod_{n} B_{n}$ is a contractive positive linear morphism. Let $\pi: \prod_{n} B_{n} \rightarrow \prod_{n} B_{n} / \bigoplus_{n} B_{n}$ be the quotient map. Then $\pi \circ \Psi$ : $C(X) \rightarrow \prod_{n} B_{n} / \bigoplus_{n} B_{n}$ is a linear map with its kernel containing $I$. Thus there is a contractive positive linear morphism $L: C(F) \rightarrow \prod_{n} B_{n} / \bigoplus_{n} B_{n}$ such that $\pi \circ \Psi=L \circ s$. It follows from [CE] that there is a contractive positive linear morphism $L_{1}: C(F) \rightarrow \prod_{n} B_{n}$ such that $\pi \circ L_{1}=L$. Write $L_{1}=\left\{\Lambda_{n}\right\}$, where $\Lambda_{n}: C(F) \rightarrow B_{n}$ are contractive positive linear morphisms. Thus, for any $f \in C(F)$,

$$
\left\|\psi_{n}(f)-\Lambda_{n}(s \circ f)\right\| \rightarrow 0 \text { as } n \rightarrow \infty .
$$

This ends the proof.

Lemma 2.3 (cf. Lemma 2.1 in [Ln7] and Lemma 2.12 in [GL2]). Let $X$ be a locally compact metric space, $G \subset X$ be an open subset,

$$
I=\left\{f \in C_{0}(X): f(x)=0 \text { if } x \notin G\right\} .
$$

For any $\varepsilon>0, \eta>0$ and a finite subset $\mathcal{F} \in C_{0}(X)$, there exist $\delta>0, \gamma>0$, $a>0$ and a finite subset $\mathcal{G} \subset C(X)$ satisfying the following: if $A$ is a $C^{*}$-algebra of real rank zero, $\phi: C_{0}(X) \rightarrow A$ is a contractive positive linear map which is $\gamma-\mathcal{G}$-multiplicative and if

$$
\left\|\phi\left(g_{a / 8} f\right)-\sum_{k=1}^{m} g_{a / 8} f\left(\xi_{k}\right) p_{k}\right\|<\delta
$$

for all $f \in \mathcal{G}$, where $\xi_{k} \in G,\left\{p_{k}\right\}$ are mutually orthogonal projections in $A$ and where $g_{\beta} \in C_{0}(X), 0 \leq g_{\beta} \leq 1, g_{\beta}(t)=0$ if dist $(t, X \backslash G)<\beta / 2$ and $g_{\beta}(t)=1$ if dist $(t, X \backslash G) \geq \beta$, if $\beta>0$, then there exists a projection $p \in A$, a contractive positive linear morphism $\Lambda: C_{0}(X \backslash G) \rightarrow p A p$ which is $\eta$-s $(\mathcal{F})$-multiplicative, finitely many points $\left\{\zeta_{k}\right\} \subset G$, and finitely many mutually orthogonal projections 
$\left\{p_{k}\right\} \subset p A p$, where $F=\{\xi \in X: \operatorname{dist}(\xi, G) \geq \sigma\}$ and $s: C_{0}(X) \rightarrow C_{0}(X \backslash G)$ is the surjective map, such that

$$
\left\|\phi(f)-\Lambda(s \circ f) \oplus \sum_{k} f\left(\zeta_{k}\right) p_{k}\right\|<\varepsilon
$$

for all $f \in \mathcal{F}$.

Proof. The proof is a modification of that of Lemma 2.11 in [GL2]. We may assume that $\mathcal{F}$ is a subset of the unit ball of $C(X)$.

Given $\eta>0$ and a finite subset $\mathcal{G}_{1}$ which is in the unit ball of $I$, there exists $a>0$ such that

$$
\left\|g_{a} f-f\right\|<\eta
$$

for all $f \in \mathcal{G}_{1} \cup \mathcal{F} \cap I$.

Set $\mathcal{G}=\mathcal{G}_{1} \cup \mathcal{F} \cup\left\{g_{a / 4}\right\}$. Suppose that

$$
\left\|\phi\left(g_{a / 8} f\right)-\sum_{k=1}^{m} g_{a / 8} f\left(\xi_{k}\right) p_{k}\right\|<\delta
$$

for all $f \in \mathcal{G}$, where $\delta>0$ will be chosen later.

Let $p=\sum_{\xi_{j} \in \Omega_{a} / 2} p_{j}$, where $\Omega_{a / 2}=\{\xi \in G: \operatorname{dist}(\xi, X \backslash G) \geq a / 2\}$. A direct computation shows, with sufficiently small $\gamma$ as well as sufficiently small $\delta$, as in the proof of Lemma 2.11 in [GL2],

$$
\left\|p \phi(f) p-\sum_{\xi_{j} \in \Omega_{a / 2}} f\left(\xi_{j}\right) p_{j}\right\|<\varepsilon / 4
$$

for all $f \in \mathcal{F}$ and

$$
\left\|(1-p) \phi\left(g_{a}\right)\right\|<\sigma
$$

for any given $\sigma>0$. We also have, with sufficiently small $\delta$ and $\eta$,

$$
\|(1-p) \phi(f)(1-p)\|<\sigma
$$

for all $f \in \mathcal{G}_{1} \cup \mathcal{F} \cap I$. Thus, by applying Lemma 2.2 , with sufficiently small $\sigma$ and sufficiently large $\mathcal{G}_{1}$, we obtain a contractive positive linear morphism $\Lambda: C(F) \rightarrow$ $(1-p) A(1-p)$ such that

$$
\|(1-p) \phi(f)(1-p)-\Lambda(s \circ f)\|<\varepsilon / 4
$$

for all $f \in \mathcal{F}$. Therefore

$$
\left\|\phi(f)-\left[\Lambda(s \circ f) \oplus \sum_{\xi_{j} \in \Omega_{a / 2}} f\left(\xi_{j}\right) p_{j}\right]\right\|<\varepsilon
$$

for all $f \in \mathcal{F}$.

Lemma 2.4. Let $X$ be a finite $C W$ complex of dimension no more than two. For any $\varepsilon>0$ and any finite subset $\mathcal{F} \in C(X)$, there exist a finite subset $\mathcal{P} \in \mathbf{P}(C(X))$, $\delta>0(\delta<\delta(\mathcal{P}))$ and a finite subset $\mathcal{G} \in C(X)$ satisfying the following: if $A \in$ $\mathbf{B}$ and $\Lambda: C(X) \rightarrow A$ is a contractive positive linear morphism which is $\delta$ - $\mathcal{G}$ multiplicative and $1 / 2 \sigma_{X, \mathcal{F}, \varepsilon / 4}-\mathcal{F}$-injective with

$$
\Lambda_{*}(\overline{\mathcal{P}})=\alpha(\overline{\mathcal{P}})
$$

for some $\alpha \in \mathcal{N} k$, then there exists a homomorphism $h: C(X) \rightarrow A$ such that

$$
\|\Lambda(f)-h(f)\|<\varepsilon
$$

for all $f \in \mathcal{F}$. 
Proof. We assume that $X$ is a polyhedron. If $X$ is a polyhedron of dimension zero, then, the lemma follows easily. The case when $X$ is a polyhedron of dimension 1 follows from Theorem 5.1 in [Lr4].

We now assume that $X$ is a finite CW complex with dimension 2. Suppose that $F \subset X$ is a one-dimensional finite $\mathrm{CW}$ complex, $\left\{Y_{i}\right\}_{i=1}^{m}$ are $m$ disks (of the plane) and $f_{i}$ are continuous functions from the boundaries of $Y_{i}$ into $F$ such that $X$ is the result of gluing $Y_{i}$ on $F$ by maps $f_{i}$.

Let $I=\{f \in C(X): f(x)=0$ for $x \in F\}$ be the ideal of $C(X)$. Since now we assume that $X$ is a finite CW complex, $K_{0}(C(X))$ is finitely generated. Let $\Lambda: C(X) \rightarrow A$ be a contractive positive linear morphism which is $\delta$ - $\mathcal{G}$-multiplicative and $\sigma$-injective, where $\delta, \mathcal{G}$ and $\sigma$ will be determined. We also assume that

$$
\Lambda_{*}(\overline{\mathcal{P}})=\alpha(\overline{\mathcal{P}})
$$

for some $\alpha \in \mathcal{N} k$ and for some finite subset $\mathcal{P} \subset \mathbf{P}(C(X))$. Let $\lambda: I \rightarrow A$ be the restriction of $\Lambda$ on $I$ and $L: C(\Omega) \rightarrow A$ be the contractive positive linear morphism induced by $\lambda$, where $C(\Omega)=\tilde{I}$. Note that $\Omega$ is the one-point compactification of the union of interior $Y_{i}$ 's. Note that $\Omega$ is the space of finitely many 2 -spheres glued at a common point. Since $K_{i}(C(\Omega))$ is finitely generated and torsion free, one computes that $K L(C(\Omega), A)=\operatorname{Hom}\left(K_{*}(C(\Omega)), K_{*}(A)\right)$. Therefore, for space $\Omega$, $\mathcal{N}_{k}=\mathcal{N}$. From $K$-theory, the natural injective map from $C(\Omega)$ into $C(X)$ induces an injective map from $\operatorname{Hom}_{(}\left(K_{0}(C(\Omega)), K_{0}(A)\right)$ into $\operatorname{Hom}_{(}\left(K_{0}(C(X)), K_{0}(A)\right)$. Let $\mathcal{Q} \subset \mathbf{P}(C(\Omega))$ be a finite subset. Then the condition that

$$
\Lambda_{*}(\overline{\mathcal{P}})=\alpha(\overline{\mathcal{P}})
$$

for some $\alpha \in \mathcal{N} k$, implies that

$$
L_{*}(\overline{\mathcal{Q}}) \in \mathcal{N},
$$

provided that $\mathcal{P}$ is sufficiently large. By Theorem 1.12, for any $\varepsilon_{1}>$ and any finite subset $\mathcal{G}_{1} \in C(\Omega)$, there is a homomorphism $h_{0}: C(\Omega) \rightarrow A$ with finite dimensional range such that

$$
\left\|L(f)-h_{0}(f)\right\|<\varepsilon_{1}
$$

for all $f \in \mathcal{G}_{1}$, provided that $\sigma$ and $\delta$ are sufficiently small and $\mathcal{G}$ is sufficiently large.

Denote by $s_{F}$ the canonical surjective map from $C(X)$ onto $C(F)$. For $\delta_{1}>$ 0 a finite subset $\mathcal{G}_{2} \in C(X)$, by applying Lemma 2.3, we obtain a projection $p \in A$, a contractive positive linear morphism $L_{0}: C(F) \rightarrow p A p$ which is $\delta_{1}$ $s_{F}(\mathcal{G})$-multiplicative and $\sigma$-injective, a finite set of mutually orthogonal projections $\left\{p_{i}\right\} \subset(1-p) A(1-p)$ and a finite set $\left\{\zeta_{i}\right\}$ in the interior of $\bigcup_{j=1}^{m} Y_{j}$ such that

$$
\left\|\Lambda(f)-\left(L_{0} \circ s_{F}(f) \oplus \sum_{i} f\left(\zeta_{i}\right) p_{i}\right)\right\|<\varepsilon_{1}
$$

for all $f \in \mathcal{G}_{2}$, provided that $\delta$ is sufficiently small and $\mathcal{G}$ is sufficiently large. Since $F$ is one-dimensional, by Theorem 5.1 in [Lr4], there is a homomorphism $h_{1}: C(F) \rightarrow p A p$ such that

$$
\left\|L_{0}(f)-h_{1}(f)\right\|<\varepsilon / 2
$$

for all $f \in s_{F}(\mathcal{F})$, provided that $\delta_{1}$ is sufficiently small and $\mathcal{G}$ is sufficiently large. We then define $h(f)=h_{1} \circ s_{F} \oplus \sum_{i} f\left(\zeta_{i}\right) p_{i}$ for $f \in C(X)$. Thus

$$
\|\Lambda(f)-h(f)\|<\varepsilon
$$


for all $f \in \mathcal{F}$, provided that $\varepsilon_{1}$ is sufficiently small (which requires that $\delta$ and $\sigma$ be small enough) and $\mathcal{G}_{2}$ is sufficiently large (which requires that $\mathcal{G}$ be large enough).

Theorem 2.5. Let $X$ be a finite $C W$ complex of dimension no more than two. For any $\varepsilon>0$ and any finite subset $\mathcal{F} \in C(X)$, there exist a finite subset $\mathcal{P} \in \mathbf{P}(C(X))$, a positive number $\delta>0(\delta<\delta(\mathcal{P}))$ and a finite subset $\mathcal{G} \in C(X)$ satisfying the following: If $A \in \mathbf{B}$ and $\Lambda: C(X) \rightarrow A$ is a contractive positive linear morphism which is $\delta$-G-multiplicative with

$$
\Lambda_{*}(\overline{\mathcal{P}})=\alpha(\overline{\mathcal{P}})
$$

for some $\alpha \in \mathcal{N} k$, then there exists an homomorphism $h: C(X) \rightarrow A$ such that

$$
\|\Lambda(f)-h(f)\|<\varepsilon
$$

for all $f \in \mathcal{F}$.

Proof. Let $\sigma=1 / 4 \sigma_{X, \mathcal{F}, \varepsilon / 4}$, where $\sigma_{X, \mathcal{F}, \varepsilon / 4}$ is as defined in Remark 1.7. Since $X$ is compact, there are compact subsets $F_{1}, F_{2}, \ldots, F_{l} \subset X$ which are themselves finite CW complex such that for any compact subset $F \subset X$, there is an $F_{j}$ with $F \subset F_{j}$ and

$$
\sup \left\{\operatorname{dist}\left(x, F_{j}\right)+\operatorname{dist}(F, y): x \in F, y \in F_{j}\right\}<\sigma .
$$

Note that $\sigma_{F_{j}, s_{j}(\mathcal{F}), \varepsilon / 4} \geq \sigma_{X, \mathcal{F}, \varepsilon / 4}$, where $s_{j}: C(X) \rightarrow C\left(F_{j}\right)$ is the surjective map. Let $\delta_{j}>0, \mathcal{P}_{j} \subset \mathbf{P}\left(C\left(F_{j}\right)\right)$ and finite subset $\mathcal{G}_{j} \subset C\left(F_{j}\right)$ be as required in Lemma 2.4 , for $\varepsilon_{1}, s_{j}(\mathcal{F})$ and space $F_{j}, j=1,2, \ldots, l$. Let $\delta^{\prime}=\min \left\{\delta_{i}: i=1,2, \ldots, l\right\}, \mathcal{G}^{\prime}=$ $\bigcup_{i} H_{i}$, where $H_{i}$ is a finite subset of $C(X)$ such that $s_{i}\left(H_{i}\right)=\mathcal{G}_{i}$. It follows from Lemma 1.17 in [Ln11] that with sufficiently large $\mathcal{G}$ and sufficiently small $\delta$, there is a compact subset $F \subset X$ and a contractive positive linear morphism $L^{\prime}: C(F) \rightarrow A$ which is $\delta^{\prime} / 2-\mathcal{G}^{\prime}$-multiplicative and $\sigma$-s $\left(\mathcal{G}^{\prime}\right)$-injective, where $s: C(X) \rightarrow C(F)$ is the surjective map, such that

$$
\left\|\psi(f)-L^{\prime}(f)\right\|<\varepsilon / 2
$$

for all $f \in \mathcal{G}^{\prime}$. Choose $F_{j}$ above so that $F \subset F_{j}$ and

$$
\sup \left\{\operatorname{dist}\left(x, F_{j}\right)+\operatorname{dist}(F, y): x \in F, y \in F_{j}\right\}<\sigma / 2 .
$$

Let $s_{0}: C\left(F_{j}\right) \rightarrow C(F)$ be the quotient map and $L=L^{\prime} \circ s_{0}: C\left(F_{j}\right) \rightarrow A$. Then $L$ is $\delta / 2-s_{j}\left(\mathcal{G}^{\prime}\right)$-multiplicative and $\sigma-s_{j}\left(\mathcal{G}^{\prime}\right)$-injective. It follows from Lemma 2.2 in [GL2] that, since $\operatorname{dim}(X) \leq 2$, there is a surjective map from $\operatorname{kerd}_{X}$ onto $\operatorname{kerd}_{F_{j}}$. Thus, with sufficiently large $\mathcal{P} \subset \mathbf{P}(C(X))$, the condition that $\Lambda_{*}(\overline{\mathcal{P}}) \in \mathcal{N}_{k}$ implies that

$$
L_{*}\left(\overline{\mathcal{P}}_{j}\right) \in \mathcal{N} k .
$$

By Lemma 2.4, there is a homomorphism $h_{1}: C\left(F_{j}\right) \rightarrow A$ such that

$$
\left\|L(f)-h_{1}(f)\right\|<\varepsilon / 2
$$

for all $f \in s_{j}(\mathcal{F})$. Note that $L^{\prime} \circ s_{0} \circ s_{j}=L \circ s^{\prime}$. We have

$$
\left\|\phi(f)-h_{1} \circ s_{j}(f)\right\|<\varepsilon
$$

for all $f \in \mathcal{F}$. Take $h=h_{1} \circ s_{j}$. 
Definition 2.6. Let $X$ be a compact metric space and $A$ be a unital $C^{*}$-algebra. Denote by $\mathcal{H}$ the subset of elements in $K L(C(X), A)$ which is represented by a homomorphism $h: C(X) \rightarrow A \otimes \mathcal{K}$. Note that if $\alpha \in \mathcal{H}$ and $\gamma$ is the map from $K L(C(X), A)$ onto $\operatorname{Hom}\left(K_{*}(C(X)), K_{*}(A)\right)$, then $\gamma(\alpha)$ preserves the order on $K_{0}(C(X))$. Fix $X$. We denote by $\mathbf{B}_{X}$ the set of those simple $C^{*}$-algebra $A \in \mathbf{B}$ satisfying the property that, for any nonzero projection $p \in A$ and $\alpha \in \mathcal{H}$, there exists a homomorphism $h: C(X) \rightarrow e A e$ such that

$$
\left.(h)_{*}\right|_{\text {kerd }}=\left.\alpha\right|_{\text {kerd }}
$$

for every compact subset of $X$. It follows from [Ln11] that every purely infinite simple $C^{*}$-algebra is in $\mathbf{B}_{X}$.

Theorem 2.7. Let $X$ be a compact metric space of dimension no more than two. For any $\varepsilon>0$ and a finite subset $\mathcal{F} \subset C(X)$, there exist a finite subset $\mathcal{G} \subset C(X)$, a positive number $\delta>0(\delta<\delta(\mathcal{P}))$ and a finite subset $\mathcal{P} \subset \mathbf{P}(C(X))$ satisfying the following: For any unital $C^{*}$-algebra $A \in \mathbf{B}_{X}$, if $\Lambda: C(X) \rightarrow A$ is a contractive positive linear morphism which is $\delta$-G-Multiplicative, $1 / 4 \sigma_{X, \mathcal{F} \varepsilon / 4}-\mathcal{F}$-injective and

$$
\Lambda_{*}=\alpha \text { on } \overline{\mathcal{P}}
$$

for some $\alpha \in \mathcal{H}$, then there exists a homomorphism $h: C(X) \rightarrow A$ such that

$$
\|\Lambda(f)-h(f)\|<\varepsilon
$$

for all $f \in \mathcal{F}$.

Proof. We first would like to point out that, in the case when $A$ is an elementary $C^{*}$ algebra, Theorem 2.7 follows from Theorem 1.12 directly. This is because $K_{1}(A)=$ 0 and $K_{0}(A)$ has no infinitesimal element and is free. Thus $\mathcal{H}=\mathcal{N}$.

So now we assume that $A$ is a nonelementary $C^{*}$-algebra among other conditions. Let $\Lambda$ be as in the theorem, with $\delta, \mathcal{G}$ and $\mathcal{P}$ to be chosen.

By Lemma 1.5 in [LP1], without loss of generality, we may write that

$$
\Lambda(f)=\sum_{i=1}^{m} f\left(\zeta_{i}\right) p_{i} \oplus \psi_{1}(f)
$$

for all $f \in C(X)$, where $\left\{\zeta_{i}\right\}$ is $2 \sigma$-dense in $X$ and $\psi_{1}(f)$ is $\delta$ - $\mathcal{G}$-multiplicative (by letting $\Lambda$ be $\delta^{\prime}-\mathcal{G}^{\prime}$-multiplicative with sufficiently small $\delta^{\prime}$ and sufficiently large $\mathcal{G}^{\prime}$ ). Since $A$ is a nonelementary simple $C^{*}$-algebra of real rank zero, there is a projection $e \in A$ such that

$$
e \oplus e \oplus e \oplus e \oplus e \preceq p_{i}
$$

for each $i$. By the assumption, for any given $\mathcal{P}$, with small enough $\delta$ and large enough $\mathcal{G}$, there is a homomorphism $\phi: C(X) \rightarrow e A e$, such that

$$
\left(\psi_{1}\right)_{* \overline{\mathcal{P}}}=\phi_{* \overline{\mathcal{P}}} .
$$

By $[\mathrm{EG}]($ note that $\operatorname{dim}(X) \leq 2)$, there is a homomorphism $\bar{\phi}: C(X) \rightarrow M_{4}(e A e)$ such that

$$
(\phi \oplus \bar{\phi})_{*} \in \mathcal{N} .
$$

Note that, since $A$ is a nonelementary simple $C^{*}$-algebra, $\phi$ (and $\bar{\phi}$ ) can always be chosen so that it is $\sigma$-injective. Now we have

$$
\left(\psi_{1} \oplus \bar{\psi}\right)_{* \overline{\mathcal{P}}}=(\phi \oplus \bar{\phi})_{* \overline{\mathcal{P}}}
$$


Applying Theorem 1.12, with sufficiently small $\delta$ and sufficiently large $\mathcal{G}$, there are homomorphisms $h_{1}: C(X) \rightarrow Q M_{5}(A) Q$ (with $Q=\operatorname{diag}\left(1-\sum_{i} p_{i}, e, e, e, e\right)$ ) and $h_{2}: C(X) \rightarrow M_{5}(e A e)$ both with finite dimensional range such that

$$
\left\|\psi_{1}(f) \oplus \bar{\phi}(f)-h_{1}(f)\right\|<\varepsilon / 4 \quad \text { and } \quad\left\|\phi(f) \oplus \bar{\phi}(f)-h_{2}(f)\right\|<\varepsilon / 4
$$

for all $f \in \mathcal{F}$. Without loss of generality (with sufficiently small $\sigma$ ), we may write $h_{2}(f)=\sum_{i=1}^{n} f\left(\zeta_{i}\right) d_{i}$, where $\left\{d_{i}\right\}$ are mutually orthogonal projections in $M_{6}(e A e)$. There is a unitary $U \in M_{7}(A)$ such that

$$
U^{*} d_{i} U \leq p_{i} \quad \text { and } \quad U\left(\sum_{i=1}^{n} p_{i}\right)=\left(\sum_{i=1}^{n} p_{i}\right) U=\left(\sum_{i=1}^{n} p_{i}\right)
$$

for $i=1,2, \ldots, n$. We estimate that

$$
\begin{aligned}
& \| \Lambda(f)-\left[\sum_{i=1}^{n} f\left(\zeta_{i}\right)\left(p_{i}-U^{*} d_{i} U\right) \oplus U^{*}\left(h_{1}(f) \oplus \phi(f)\right) U\right] \| \\
& \leq\left\|\psi(f)-\left[\sum_{i=1}^{n} f\left(\zeta_{i}\right)\left(p_{i}-U^{*} d_{i} U\right) \oplus \phi_{1}(f) \oplus U^{*} h_{2}(f) U\right]\right\| \\
&+\|\left[\sum_{i=1}^{n} f\left(\zeta_{i}\right)\left(p_{i}-U^{*} d_{i} U\right) \oplus \phi_{1}(f) \oplus U^{*} h_{2}(f) U-U^{*}\left(h_{1} \oplus \phi(f)\right) U \|\right. \\
&<\varepsilon / 4+\varepsilon / 4<\varepsilon
\end{aligned}
$$

for all $f \in \mathcal{F}$.

Remark 2.8. Note as in Remark 1.13 that if $K_{i}(C(X))$ is torsion free $(i=0,1)$, then we only need to consider projections in $\bigcup_{m} M_{m}(C(X)) \oplus M_{m}\left(C(X) \otimes C\left(S^{1}\right)\right)$. Suppose that $A \in \mathbf{B}$ such that $K_{0}(A)$ is a dimension group. Suppose that $B$ is a (simple) AF-algebra with $K_{0}(A)=K_{0}(B)$ and $\alpha \in \mathcal{H}$. A result in [Li] says that, for any nonzero projection $p \in B$, there is a homomorphism $h: C(X) \rightarrow p B p$ with $\left.h_{*}\right|_{k e r d_{X}}=\left.\alpha\right|_{k e r d_{X}}$. It follows from 2.9 in [Ln1] that there is a unital inclusion $j: B \rightarrow A$ such that $j$ induces an isomorphism from $K_{0}(B)$ onto $K_{0}(A)$. This implies that $A \in \mathbf{B}_{X}$. Now let $X$ be a compact metric space of dimension no more than 2. Then, in 2.7, the condition that $A \in \mathbf{B}_{X}$ can be replaced by $K_{0}(A)$ is a dimension group. More significantly, the injective condition can be removed. This is because if $F \subset X$, the surjection maps $k e r d_{X}$ onto $k e r d_{F}$. Thus, one can combine the proof of 2.5 with the proof of 2.7 to get the following corollary. Note that the condition that $\Lambda_{*}=\alpha$ on $\overline{\mathcal{P}}$ for some $\alpha \in \mathcal{H}$ is necessary.

Corollary 2.9. Let $X$ be a compact metric space of dimension no more than two. For any $\varepsilon>0$ and a finite subset $\mathcal{F} \subset C(X)$, there exist a finite subset $\mathcal{G} \subset C(X)$, a positive number $\delta>0(\delta<\delta(\mathcal{P}))$ and a finite subset $\mathcal{P} \subset \mathbf{P}(C(X))$ satisfying the following: For any unital $C^{*}$-algebra $A \in \mathbf{B}$ with $K_{0}(A)$ being a dimension group, if $\Lambda: C(X) \rightarrow A$ is a contractive positive linear contractive positive linear morphism which is $\delta$-G-multiplicative and

$$
\Lambda_{*}=\alpha \text { on } \overline{\mathcal{P}}
$$

for some $\alpha \in \mathcal{H}$, then there exists a homomorphism $h: C(X) \rightarrow A$ such that

$$
\|\Lambda(f)-h(f)\|<\varepsilon
$$

for all $f \in \mathcal{F}$. 
2.10. Now we give a very special example. Let

$$
\begin{gathered}
f\left(e^{2 \pi i t}\right)= \begin{cases}1-2 t, & \text { if } 0 \leq t \leq 1 / 2, \\
-1+2 t, & \text { if } 1 / 2 \leq t \leq 1,\end{cases} \\
g\left(e^{2 \pi i t}\right)= \begin{cases}\left(f(t)-f(t)^{2}\right)^{1 / 2}, & \text { if } 0 \leq t \leq 1 / 2, \\
0, & \text { if } 1 / 2 \leq t \leq 1,\end{cases} \\
h\left(e^{2 \pi i t}\right)= \begin{cases}0, & \text { if } 0 \leq t \leq 1 / 2, \\
\left(f(t)-f(t)^{2}\right)^{1 / 2}, & \text { if } 1 / 2 \leq t \leq 1 .\end{cases}
\end{gathered}
$$

Let $A$ be a (unital) $C^{*}$-algebra. For any pair of unitaries $u$ and $v$ in $A$, define

$$
e(u, v)=\left(\begin{array}{cc}
f(v) & g(v)+h(v) u^{*} \\
g(v)+u h(v) & 1-f(v)
\end{array}\right),
$$

where $f, g$ and $h$ are as above. If $u$ commutes with $v$, then $e(u, v)$ is a projection. In the general case, $e(u, v)$ is always selfadjoint. If $\|u v-v u\|$ is small, then

$$
\left\|e(u, v)^{2}-e(u, v)\right\|
$$

is small (see [Lr1, 3.5 and 3.6]). There is a positive number $\delta_{0}$ (which is independent of $A, u$ and $v$ ) such that whenever

$$
\|u v-v u\|<\delta_{0}
$$

then

$$
s p(e(u, v)) \subset[-1 / 4,1 / 4] \cup[3 / 4,1+1 / 4]
$$

(see $[\operatorname{Lr} 1,3.5$ and 3.6]). Let $\chi$ be the characteristic function for the subset $[1 / 2,3 / 2]$. Then $\chi(e(u, v))$ is a projection in the $C^{*}$-subalgebra of $A$ generated by $e(u, v)$ and

$$
\|\chi(e(u, v))-e(u, v)\| \leq 1 / 4 .
$$

The Exel-Loring index (cf. [EL2]) $\kappa(u, v)$ is defined by

$$
\kappa(u, v)=[\chi(e(u, v))]-\left[\left(\begin{array}{ll}
1 & 0 \\
0 & 0
\end{array}\right)\right] \quad \text { in } K_{0}(A) .
$$

Let $A$ be a $C^{*}$-algebra in $\mathbf{B}$ which is not elementary. It is known and (easy to show) that for any $\alpha_{1} \in \operatorname{Hom}\left(K_{1}\left(C\left(\mathbf{T}^{2}\right)\right), K_{1}(A)\right)$, there is a homomorphism $h_{1}: C\left(\mathbf{T}^{2}\right) \rightarrow e A e$ for any nonzero projection $e \in A$ such that $h_{1}$ induces $\alpha_{1}$. Thus if $\alpha \in \operatorname{Hom}\left(K_{*}\left(C\left(\mathbf{T}^{2}\right)\right), K_{*}(A)\right)$ with $\left.\alpha\right|_{\operatorname{kerd}_{\mathbf{T}^{2}}}=0$ and $\alpha\left(\left[1_{C\left(\mathbf{T}^{2}\right)}\right]\right)=\left[1_{A}\right]$, then there is a unital homomorphism $h: C\left(\mathbf{T}^{2}\right) \rightarrow A$ such that $[h]=\alpha$. If $K_{0}(A)$ is a dimension group, then, by a result in [Li], $\alpha \in \mathcal{H}$ if and only if $\left.\alpha\right|_{\text {kerd }_{\mathbf{T}^{2}}}$ are infinitesimal elements in $K_{0}(A)$, or equivalently, $t(\alpha(x))=0$ for each $x \in \operatorname{kerd}_{\mathbf{T}^{2}}$ and all normalized quasitraces of $A$.

Corollary 2.11 (cf. [Lr5]). For any $\varepsilon>0$, there is $\delta>0$ so that, whenever $A \in \mathbf{B}$, if $u$ and $v$ are two unitaries in $A$,

$$
\|u v-v u\|<\delta \quad \text { and } \quad \kappa(u, v)=0,
$$

then there exist commuting unitaries $u_{1}, v_{1} \in A$ such that

$$
\left\|u-u_{1}\right\|<\varepsilon \text { and }\left\|v-v_{1}\right\|<\varepsilon .
$$

Furthermore,

(a) if $K_{1}(A)=0, u_{1}$ and $v_{1}$ can be required to have finite spectrum; 
(b) if $A$ is a purely infinite simple $C^{*}$-algebra, the condition that $\kappa(u, v)=0$ is not needed;

(c) if $K_{0}(A)$ is a dimension group, the condition that $\kappa(u, v)=0$ can be replaced by $\tau(\kappa(u, v))=0$ for all normalized quasitraces $\tau$ of $A$.

Proof. (See the proof of 3.21 in [GL2].) Define two homomorphisms $h_{1}, h_{2}: C\left(S^{1}\right) \rightarrow$ $A$ by the unitaries $u$ and $v$ in $A$. It follows from Lemma 2.1 in [LP1] that, for any $\varepsilon_{1}>0$ and any finite subset $\mathcal{F}_{1} \in C\left(\mathbf{T}^{2}\right)$ there exists a contractive positive linear map $L: C\left(\mathbf{T}^{2}\right) \rightarrow A$ which is $\varepsilon_{1}-\mathcal{F}_{1}$-multiplicative such that

$$
\left\|L\left(z_{1}\right)-u\right\|<\varepsilon \quad \text { and } \quad\left\|L\left(z_{2}\right)-v\right\|<\varepsilon \text {, }
$$

where $z_{1}$ and $z_{2}$ are standard unitary generators of $C\left(\mathbf{T}^{2}\right)$. So, without loss of generality, we may assume that $L\left(z_{1}\right)=u$ and $L\left(z_{2}\right)=v$. Therefore the first part of the corollary follows from the last part of Corollary 2.11 and Theorem 2.5 (and Remark 2.8).

Part (a) is clear. Part (b) follows from [Ln5]. Part (c) follows from 2.10 and the last part of Corollary 2.11.

We note that, in the case when $K_{0}(A)$ is a dimension group, the condition $\tau(\kappa(u, v))=0$ is also necessary.

\section{REFERENCES}

[BkD] I. D. Berg and K. Davidson, Almost commuting matrices and a quantitative version of Brown -Douglas-Fillmore theorem, Acta Math., 166 (1991), 121-161. MR 92f:47015

[Bl1] B. Blackadar, K-Theory for Operator Algebras, Springer-Verlag, New York, Berlin, 1986. MR 88g:46082

[Bn1] L. G. Brown, Stable isomorphism of hereditary subalgebras of $C^{*}$-algebras, Pacific J. Math., 71 (1977), 335-348. MR 56:12894

[Bn2] L. G. Brown, Interpolation by projections in $C^{*}$-algebras of real rank zero, J. Operator Theory, 71 (1977), 335-348.

[BDF1] L. G. Brown, R. Douglas, and P. Fillmore, Unitary equivalence modulo the compact operators and extensions of $C^{*}$-algebras, Proc. Conf. on Operator Theory, Springer Lecture Notes in Math. 345 (1973), 56-128. MR 52:1378

[BDF2] L. G. Brown, R. Douglas, and P. Fillmore, Extensions of $C^{*}$-algebras and $K$-homology, Ann. of Math. 105 (1977), 265-324. MR 56:16399

[BP1] L. G. Brown and G. K. Pedersen, $C^{*}$-algebras of real rank zero, J. Funct. Anal. 99(1991), 131-149. MR 92m:46086

[BP2] L. G. Brown and G. K. Pedersen, On the geometry of the unit ball of a $C^{*}$-algebra, part II , preprint of Mathematics Institute of Copenhagen University (1993).

[BP3] L. G. Brown and G. K. Pedersen, On the geometry of the unit ball of a $C^{*}$-algebra, J. Reine Angew. Math. 469(1995), 113-147. MR 96m:46101

[Ch] M. D. Choi, Almost commuting matrices need not be nearly commuting, Proc. Amer. Math. Soc. 102 (1988), 529-609. MR 89b:47021

[CE] M-D. Choi and E. Effros, The completely positive lifting problem for $C^{*}$-algebras, Ann. of Math. 104 (1976), 585-609. MR 54:5843

[Cu] J. Cuntz, K-theory for certain $C^{*}$-algebras, Ann. of Math. 113 (1981), 181-197. MR 84c: 46058

[D1] M. Dadarlat, Reduction to dimension three of local spectra of real rank zero $C^{*}$-algebras, J. Reine Angew. Math., 460 (1995), 189-212. MR 95m:46116

[D2] M. Dadarlat, Approximately unitarily equivalent morphisms and inductive limit $C^{*}$ algebras, K-Theory, 9 (1995), 117-137. MR 96h:46088

[DL1] M. Dadarlat and T. Loring, The K-theory of abelian subalgebras of AF-algebras, J. Reine Angew. Math. 432 (1992), 39-55. MR 94b:46099

[DL2] M. Dadarlat and T. Loring, K-homology, asymptotic representations, and unsuspended E-theory J. Funct. Anal. 129 (1994), 367-384. MR 96d:46092 
[DL3] M. Dadarlat and T. Loring, A universal multi-coefficient theorem for the Kasparov groups, Duke Math. J., 84 (1996), 355-377. MR 97f:46109

[D] K. Davidson, Almost commuting hermitian matrices, Math. Scand. 56 (1985), 222-240. MR 87e:47012

[DN] M. Dadarlat and A. Nemethi, Shape theory and connected K-theory, J. Operator Theory 23 (1990), 207-291. MR 91j:46092

[Eff] E. Effros, Dimensions and $C^{*}$-algebras, CBMS Regional Conf. Ser. in Math., no. 46, Amer. Math. Soc., Providence, RI, 1981. MR 84k:46042

[El11] G. A. Elliott, On the classification of $C^{*}$-algebras of real rank zero, J. Reine Angew. Math. 443 (1993), 179-219. MR 94i:46074

[El12] G. A. Elliott, A classification of certain simple $C^{*}$-algebras, pp 373-385 in: Quantum and Non-Commutative Analysis (edited by H. Araki et al.), Kluwer, Dordrecht, 1993. MR 95h: 46089

[El13] G. A. Elliott, Are amenable $C^{*}$-algebras classifiable?, Representation Theory of Groups and Algebras, Contemporary Math. vol 145, Amer. Math. Soc., Providence, RI, 1993. CMP 93:11

[El14] G. A. Elliott, The classification problem for amenable $C^{*}$-algebras, Proc. ICM Vol. 1, 2 (Zurich, 1994), Birkhauser, Basel, 1995, 922-932. MR 97g:46072

[EG] G. A. Elliott and G. Gong, On the classification of $C^{*}$-algebras of real rank zero, II, Ann. Math., 144 (1996), 497-610. CMP 97:06

[EGLP] G. A. Elliott, G. Gong, H. Lin and C. Pasnicu, Abelian $C^{*}$-subalgebras of $C^{*}$-algebras of real rank zero and inductive limit $C^{*}$-algebras, Duke Math. J., 85, (1996) 511-554. MR 98a: 46076

[EgL] G. A. Elliott and T. Loring, AF-embeddings of $C\left(T^{2}\right)$ with prescribed $K$-theory, J. Funct. Anal. 103 (1992), 1-25. MR 93b:46134

[ER] G. A. Elliott and M. Rørdam, Classification of certain infinite simple $C^{*}$-algebras, II, Comment. Math. Helv., 70 (1995), 615-638. MR 96e:46080b

[Ex] R. Exel, A Fredholm operator approach to Morita equivalence, K-Theory, 7 (1993), 285-308. MR 94h:46107

[EL1] R. Exel and T. A. Loring, Almost commuting unitary matrices, Proc. Amer. Math. Soc., 106 (1989), 913-915. MR 89m: 15003

[EL2] R. Exel and T. A. Loring, Invariants of almost commuting unitaries, J. Funct. Anal. 95 (1991), 364-376. MR 92a:46083

[FR] P. Friis and M. Rørdam, Almost commutative self-adjoint matrices-a short proof of Huaxin Lin's theorem, J. Reine Angew. Math., 479 (1996), 121-131. MR 97i:46097

[G1] G. Gong, Classification of $C^{*}$-algebras of real rank zero and unsuspended E-equivalent types, J. Funct. Anal., 152 (1998), 281-329. CMP 98:09

[G2] G. Gong, On inductive limits of matrix algebras over higher dimensional spaces, part I, II, Math. Scand., 80 (1997), 41-55, 56-100. CMP 97:17

[G4] G. Gong, Approximation by dimension drop algebras and classification, C. R. Math. Rep. Acad. Sci. Canada, 16 (1994), no.1, 40-44. MR 95c:46119

[GL1] G. Gong and H. Lin, Classification of homomorphisms from $C(X)$ into a simple $C^{*}$-algebra of real rank zero, preprint.

[GL2] G. Gong and H. Lin, Almost multiplicative morphisms and almost commutative matrices, preprint.

[Gd] K. R. Goodearl, Notes on a class of simple $C^{*}$-algebras with real rank zero, Publ. Math., 36 (1992), 637-654. MR 94f:46092

$[\mathrm{K}] \quad$ E. Kirchberg, in preparation.

[Li] L. Li, $C^{*}$-algebra homomorphisms and $K K$-theory, preprint.

[Ln1] H. Lin, Skeleton $C^{*}$-subalgebras, Canad. J. Math., 44 (1992), 324-341. MR 93h:46080

[Ln2] H. Lin, $C^{*}$-algebra Extensions of $C(X)$, Memoirs Amer. Math. Soc., 115 (1995), no. 550. MR 96b: 46098

[Ln3] H. Lin, Approximation by normal elements with finite spectra in $C^{*}$-algebras of real rank zero, Pacific. J. Math., 173 (1996), 443-489. CMP 96:14

[Ln4] H. Lin, Almost commuting unitaries and classification of purely infinite simple $C^{*}$ algebras, J. Funct. Anal., to appear.

[Ln5] H. Lin, Almost commuting unitaries in purely infinite simple $C^{*}$-algebras, Math. Ann., 303 (1995), 599-616. MR 96k:46101

[Ln6] H. Lin, Almost normal elements, Inter. J. Math., 5 (1994), 765-778. MR 95f:46096 
[Ln7] H. Lin, Almost commuting selfadjoint matrices and applications, The Fields Institute Communication, 13 (1997), 193-234. MR 98c:46121

[Ln8] H. Lin, Extensions by $C^{*}$-algebras with real rank zero, Inter. J. Math., 4 (1993) 231-252. MR 94h:46095

[Ln9] H. Lin, Extensions by $C^{*}$-algebras with real rank zero II, Proc. London Math. Soc., 71 (1995), 641-674. MR 96j:46072

[Ln10] H. Lin, Homomorphisms from $C(X)$ into $C^{*}$-algebras, Canad. J. Math., 49 (1997), 9631009. CMP 98:08

[Ln11] H. Lin, Almost multiplicative morphisms and some applications, J. Operator Theory, $\mathbf{3 7}$ (1997), 121-154. MR 98b:46091

[Ln12] H. Lin, $C^{*}$-algebras with weak (FN), J. Funct. Anal., 150 (1997), 65-74. CMP 98:02

[Ln13] H. Lin, Extensions of $C(X)$ by simple $C^{*}$-algebras of real rank zero, Amer. J. Math., 119 (1997), 1263-1289. CMP 98:04

[LP1] H. Lin and N. C. Phillips, Almost multiplicative morphisms and Cuntz-algebra $\mathrm{O}_{2}$, J. International Math., 6 (1995) 625-643. MR 97h:46097

[LP2] H. Lin and N. C. Phillips, Approximate unitary equivalence of homomorphisms from $\mathcal{O}_{\infty}$, J. Reine Angew. Math. 464 (1995), 173-186. MR 98c:46120

[Lr1] T. A. Loring, $C^{*}$-algebras generated by stable relations, J. Funct. Anal. 112 (1993), 159203. MR 94k:46115

[Lr2] T. A. Loring, K-theory and asymptotically commuting matrices, Canad. J. Math. 40 (1988), 197-216. MR 89b:47022

[Lr3] T. A. Loring, Normal elements of $C^{*}$-algebras of real rank zero without finite-spectrum approximants, Proc. London Math. Soc. 51 (1995), 353-364. MR 96b:46099

[Lr4] T. A. Loring, Stable relations II: corona semiprojectivity and dimension-drop $C^{*}$-algebras, Pacific J. Math., 172 (1996), 461-475. MR 97c:46070

[Lr5] T. A. Loring, When matrices commute, preprint.

[M] S. Mardesic, On covering dimension and inverse limits of compact spaces, Illinois J. Math 4 (1960), 278-291. MR 22:7101

[Pd] G. K. Pedersen, $S A W^{*}$-algebras and corona $C^{*}$-algebras, contribution to non-commutative topology, J. Operator Theory 15 (1986), 15-32. MR 87a:46095

[Ph1] N. C. Phillips, Approximate unitary equivalence of homomorphisms from odd Cuntz algebras, Fields Inst. Commun, 13, Amer. Math. Soc., Providence, RI, 1997. MR 97k:46068

[Ph2] N. C. Phillips, A Classification theorem for nuclear purely infinite simple $C^{*}$-algebras, preprint 1995.

[Rf] M. Rieffel, Dimension and stable rank in the $K$-theory of $C^{*}$-algebras, Proc. London Math. Soc. 46 (1983), 301-333. MR 84g:46085

[Rr1] M. Rørdam, Classification of certain infinite simple $C^{*}$-algebras, J. Funct. Anal. 131 (1995), 415-458. MR 96e:46080a

[Rr2] M. Rørdam, Classification of certain infinite simple $C^{*}$-algebras, III, Fields Inst. Commun., 13, Amer. Math. Soc., Providence, RI, 1997. CMP 97:05

[RS] J. Rosenberg and C. Schochet, The Künneth theorem and the universal coefficient theorem for Kasparov's generalized K-functor, Duke Math. J. 55(1987), 431-474. MR 88i:46091

[Sch] C. Schochet, Topological methods for $C^{*}$-algebras IV: $\bmod p$ homology, Pacific J. Math., 114 (1984), 447-468. MR 86g:46103

[V] D. Voiculescu, A non-commutative Weyl-von Neumann Theorem, Rev. Roum. Math. Pures et Appl. 21 (1976), 97-113. MR 54:3427

[Zh1] S. Zhang, $C^{*}$-algebras with real rank zero and the internal structure of their corona and multiplier algebras, Part I, Pacific J. Math., 155(1992), 169-197. MR 94i:46093

[Zh2] S. Zhang, $C^{*}$-algebras with real rank zero and the internal structure of their corona and multiplier algebras, Part III, Canad. J. Math. 42 (1990), 159-190. MR 94i:46095

[Zh3] S. Zhang, K1-groups, quasidiagonality, and interpolation by multiplier projections, Trans. Amer. Math. Soc. 325 (1991), 793-818. MR 91j:46069

[Zh4] S. Zhang, Certain $C^{*}$-algebras with real rank zero and their corona and multiplier algebras, II, K-Theory, 6 (1992), 1-27. MR 94i:46094

Department of Mathematics, East China Normal University, Shanghai 200062, China Current address: Department of Mathematics, University of Oregon, Eugene, Oregon 974031222

E-mail address: hlin@darkwing.uoregon.edu 